Research Paper

\title{
Type-IV Antifreeze Proteins are Essential for Epiboly and Convergence in Gastrulation of Zebrafish Embryos
}

\author{
Qing Xiao*, Jian-Hong Xia*,Xiao-Juan Zhang, Zhi Li, Yang Wang, Li Zhou, Jian-Fang Gui ${ }^{\bowtie}$ \\ State Key Laboratory of Freshwater Ecology and Biotechnology, Institute of Hydrobiology, Chinese Academy of Sciences, University of the \\ Chinese Academy of Sciences, Wuhan 430072, China. \\ *These authors contributed equally to this work.
}

$\triangle$ Corresponding author: Tel: +86-27-68780707; Fax: +86-27-68780123; E-mail: jfgui@ihb.ac.cn.

(C) Ivyspring International Publisher. This is an open-access article distributed under the terms of the Creative Commons License (http://creativecommons.org/ licenses/by-nc-nd/3.0/). Reproduction is permitted for personal, noncommercial use, provided that the article is in whole, unmodified, and properly cited.

Received: 2014.03.16; Accepted: 2014.05.18; Published: 2014.06.24

\begin{abstract}
Many organisms in extremely cold environments such as the Antarctic Pole have evolved antifreeze molecules to prevent ice formation. There are four types of antifreeze proteins (AFPs). Type-IV antifreeze proteins (AFP4s) are present also in certain temperate and even tropical fish, which has raised a question as to whether these AFP4s have important functions in addition to antifreeze activity. Here we report the identification and functional analyses of AFP4s in cyprinid fish. Two genes, namely $a f p 4 a$ and $a f p 4 b$ coding for AFP4s, were identified in gibel carp (Carassius auratus gibelio) and zebrafish (Danio rerio). In both species, afp $4 a$ and afp $4 b$ display a head-to-tail tandem arrangement and share a common 4-exonic gene structure. In zebrafish, both afp $4 a$ and $a f p 4 b$ were found to express specifically in the yolk syncytial layer (YSL). Interestingly, afp $4 a$ expression continues in YSL and digestive system from early embryos to adults, whereas afp $4 b$ expression is restricted to embryogenesis. Importantly, we have shown by using afp $4 a$-specific and $a f p 4 b$-specifc morpholino knockdown and cell lineage tracing approaches that AFP4a participates in epiboly progression by stabilizing yolk cytoplasmic layer microtubules, and AFP4b is primarily related to convergence movement. Therefore, both AFP4 proteins are essential for gastrulation of zebrafish embryos. Our current results provide first evidence that AFP such as AFP4 has important roles in regulating developmental processes besides its well-known function as antifreeze factors.
\end{abstract}

Key words: antifreeze protein; yolk syncytial layer; epiboly; convergence; morphogenesis.

\section{Introduction}

During vertebrate embryogenesis, embryonic cell movements are very critical for morphogenesis and establishment of normal embryo architecture [1]. Through this process, the blastoderm transforms into three germ layers of endoderm, mesoderm and ectoderm as well as two major body axes of anterior-posterior axis and ventral-dorsal axis, and these changes mainly result from the harmonized morphogenetic movements, including epiboly, involution, convergence and extension (CE) [2, 3]. Numerous studies have shed light on some signaling molecules $[3,4]$, and some key factors that are related to cell adhesion, cytoskeletal rearrangement and cell inter- actions between the enveloping layer (EVL) and the yolk syncytial layer (YSL) have been suggested to play significant roles in gastrulation cell movements and embryonic development [5]. For example, the lipid pregnenolone, produced from cholesterol by Cyp11a1 enzyme, was shown to promote zebrafish (Danio rerio, Dr) embryonic cell movements by increasing yolk cytoplasmic layer (YCL) microtubule abundance [6]. In addition, Npc1 (Niemann-Pick disease, type $\mathrm{C} 1$ ), a 13 transmembrane-spanning protein containing a sterol-sensing domain, was also demonstrated to contribute early morphogenetic movements including epiboly and CE movements [7]. Neverthe- 
less, most of the significant regulators remain to be further identified because embryonic cell movements have been believed to be driven by a variety of cellular behaviors and other molecules [8].

Following our systematic studies on maternal factor screening and gene function identification [9-13] in polyploid gibel carp (Carassius auratus gibelio, Cag) with multiple modes of unisexual and sexual reproduction [14-19], we have also identified and characterized a type-IV antifreeze protein gene (afp4) [20]. Antifreeze proteins (AFPs) have been found in fungi, bacteria, plants and animals, and they can bind to ice crystals to inhibit growth and recrystallization of ice [21]. According to their molecular structures, four types of AFPs, type I-IV AFPs, have been characterized in teleost fishes [22]. The type-IV antifreeze protein (AFP4) was firstly discovered from longhorn sculpin (Myoxocephalus octodecimspinosis) in 1997 [23], and its homologues had been identified in other teleost fishes, ranging from polar to tropic region and from seawater to freshwater [20, 24, 25]. As a new type of AFP, their molecular structures have been characterized by the conserved four-helix bundle [24-26], and the antifreeze activity has been demonstrated in several fishes [23-26]. Significantly, along with wide discoveries of afp 4 homologues, their abundant expression has been detected recently in oocytes [27, 28] and in embryos [20] from some teleost fishes. So far, AFPs have been studied for more than 30 years, but almost all the previous studies have focused on the structural and biochemical properties in freezing avoidance [29-31], and little is known about the biological functions in embryonic development. The finding about abundant expression of afp 4 during embryogenesis in gibel carp provides a good chance for us to explore the biological roles. In this study, we firstly identify two tandem duplicated afp4 genes from gibel carp and zebrafish, analyze their genomic organization, and characterize their expression pattern. Then, we use zebrafish as a model to reveal their biological functions as two key regulators in early morphogenetic movements of zebrafish embryogenesis.

\section{Materials and Methods}

\section{Full-length cDNA cloning}

A positive BAC clone of afp 4 was isolated from gibel carp BAC library [32] by PCR screening based on the cDNA sequence of afp4 in gibel carp (Cagafp4, GenBank accession No. AY365004) and it was sequenced as described previously [10]. Zebrafish database search was performed on the web server of NCBI by BLAST using the CDNA of Cagafp4 as a query of nucleotide collection (nr/nt) database.

To achieve full-length cDNA sequence of the other Cagafp4 (Cagafp4a) and reexamine those of afp $4 \mathrm{~s}$ in zebrafish (Drafp4s), total RNAs of gibel carp or zebrafish embryos were purified with TRIzol Reagent (Ambion, USA) as described previously [33], and then SMART cDNA libraries were constructed according to the SMARTer ${ }^{\mathrm{TM}}$ PCR cDNA Synthesis Kit User Manual (Clontech, USA). The Cagafp $4 a$ and Drafp 4 s cDNAs were completed by $3^{\prime}$ and $5^{\prime}$ rapid amplification of cDNA ends (RACE), with primers (Table 1) based on the obtained Cagafp4s or Drafp4s cDNA sequences. The complete cDNA sequences of Cagafp $4 a$ and Drafp $4 a$ were deposited in GenBank (accession No. KJ183062 and KJ183061, respectively).

\section{Sequence and phylogenetic analyses}

The genomic structures of Cagafp4s and Drafp4s were achieved by comparative analyses between cDNA sequences and corresponding genomic sequences.

The complete sequences of other functionally characterized AFP4s in Myoxocephalus octodecemspinosus $(\mathrm{Mo})[23,26]$, Pleuragramma antarcticum $(\mathrm{Pa})$, Notothenia coriiceps $(\mathrm{Nc})$ [25] and Gadus morhua (Gm) [27, 28] were downloaded from GenBank non-redundant protein database. An unrooted maximum likelihood (ML) phylogenetic tree of AFP4s was constructed by MEGA 6 [34] with 1000 bootstrap replications, in which their evolutionary distances were computed using the JTT+F model, which was selected by ProtTest 2.4 [35] based on the corrected Akaike information criterion. Multiple amino acid sequence alignment was performed by Clustalx program (EMBL-EBI, UK). Signal peptides were predicted by a web tool SignalP 3.0 Server (CBS, Denmark). Identities and similarities between DrAFP4a and the other seven AFP4s were acquired by pairwise alignment using a web tool EMBOSS Needle (EMBL-EBI, UK). Sequence alignment of Drafp $4 a$ and Drafp $4 b$ cDNA was performed by DNAMAN 6.0 software (Lynnon Biosoft, Canada).

\section{Zebrafish manipulation}

Wild type (WT) zebrafish were maintained as described by Westerfield, and all the zebrafish embryos were obtained from natural spawning, and rinsed with embryos medium [36]. Embryos were incubated at $28.5^{\circ} \mathrm{C}$ and staged according to Kimmel et al [2]. The animal protocol for this research was approved by the Institute of Hydrobiology Institutional Animal Care and Use Committee (Approval ID: keshuizhuan 0829). 
Table I. Primers used in this study.

\begin{tabular}{|c|c|c|}
\hline Name & Sequence $\left(5^{\prime} \rightarrow 3^{\prime}\right)$ & Usage \\
\hline Cagafp4a-5'Race & AACCAGCATACATACGAAGAG & RACE \\
\hline Cagafp $4 a-3$ 'Race & TTCCTCATCGCTGTCCTTGTTAC & \\
\hline Drafp $4 a-5$ 'Race & CAGAGGCTTGATCTGCTCT & \\
\hline Drafp $4 a-3$ 'Race & CAATCTCTGGACGCAAGG & \\
\hline Drafp $4 b-5$ 'Race & GGCAATAGGTTTAATCTGGTCC & \\
\hline Drafp $4 b-3$ 'Race & TATCGTCACATTGACACAAGG & \\
\hline 5' PCR Primer & AAGCAGTGGTATCAACGCAGAGTAC & \\
\hline 3' PCR Primer & AAGCAGTGGTATCAACGCAGAGTACTTTTT & \\
\hline Dr $\beta$-actin-F & AGCACGGTATTGTGACTAACTG & qPCR and \\
\hline Dr $\beta$-actin-R & TCGAACATGATCTGTGTCATC & semi-quantitative RT-PCR \\
\hline Drafp $4 a-\mathrm{RT}-\mathrm{F}$ & CAATCTCTGGACGCAAGG & \\
\hline Drafp $4 a-\mathrm{RT}-\mathrm{R}$ & CAGAGGCTTGATCTGCTCT & \\
\hline Drafp $4 b$-RT-F & TATCGTCACATTGACACAAGG & \\
\hline Drafp $4 b-\mathrm{RT}-\mathrm{R}$ & GGCAATAGGTTTAATCTGGTCC & \\
\hline Drafp $4 a-W I S H-F$ & TCTGGACGCAAGGCAACT & WISH \\
\hline Drafp $4 a-W I S H-R$ & TCTAATACGACTCACTATAGGGAAACAAAACCATCAGCATAC & \\
\hline Drafp $4 b$-WISH-F & AGAGCCCAGTTTCAGCCCAT & \\
\hline Drafp $4 b$-WISH-R & TAATACGACTCACTATAGGGACAATCAACTCCAAAATATCAGG & \\
\hline Drafp $4 a-E G F P-F$ & GGATCCTCTGGACGCAAGGCAACT & Synthesizing afp 4:EGFP \\
\hline Drafp $4 a-E G F P-R$ & AAGCTTTGGCCTGGTCAGCAACAAAT & mRNA \\
\hline Drafp $4 b-E G F P-F$ & GGATCCGACACACCATCACACGACA & \\
\hline Drafp $4 b-E G F P-R$ & AAGCTTCTCTGCTCTCCTCCAGGTAAG & \\
\hline EGFP-F & AAGCTTATGGTGAGCAAGGGCGA & \\
\hline EGFP-R & CTCGAG TTACTTGTACAGCTCGTCCAT & \\
\hline Drafp $4 a-\mathrm{F}$ & TGGATCCATGAAATTCTCCCTCATCG & Synthesizing afp 4 mRNA \\
\hline Drafp $4 a-\mathrm{R}$ & CGCTCGAGAAACAAAACCATCAGCATAC & \\
\hline Drafp $4 b-\mathrm{F}$ & TGGATCCATGAAACTCTCССТСATC & \\
\hline Drafp $4 b-\mathrm{R}$ & CGCTCGAGGGATACTCATATCTGGAG & \\
\hline
\end{tabular}

\section{RNA isolation, real-time PCR and semi-quantitative $\mathbf{R T}$-PCR}

Total RNAs were isolated from zebrafish embryos or tissues of adults using TRIzol Reagent as described previously [33], and cDNAs were then generated using M-MLV Reverse Transcriptase (Promega, USA) as described previously [37]. Real-time PCR (qPCR) and semi-quantitative RT-PCR analyses were performed with specific primers for $a f p 4 a, a f p 4 b$ and $\beta$-actin (Table 1 ) as described previously $[37,38]$.

\section{Western blot analyses}

Western blot analyses were performed as reported previously using the anti-CagAfp4 antibody (1:200) [20]. Immunoreactive bands were visualized by using BCIP/NBT staining.

\section{Whole-mount in situ hybridization}

Whole-mount in situ hybridization (WISH) was carried out as previously described [39]. For antisense probe synthesis, T7 RNA polymerase promoter was added to the 5 ' end of reverse primers and a DIG RNA labeling kit (Roche, Germany) was used. In brief,
DNA templates of Drafp $4 a / b$ were amplified by RT-PCR from zebrafish embryos cDNA with the primers Drafp $4 a / b$-WISH-F and Drafp $4 a / b$-WISH-R (Table 1). The riboprobe produced for Drafp $4 a$ was targeted to nucleotides 20-525 (accession No. KJ183061) and for Drafp $4 b$ to nucleotides 292-844 (accession No. BC153962). In addition, antisense probes of the following mRNAs were synthesized and used: foxA3, gsc, ntl, myoD, hgg1, dxl3b, pax2.1, sox17, eve1. For sectioning, $15 \mu \mathrm{m}$ sections of the stained shield to bud stage embryos were made on a CM 1850 frozen microtomy (Leica, Germany) according to the previous report [40]. Images were acquired by Leica S8APO dissecting microscope (Leica, Germany). To measure the extent of epiboly or mediolateral length of somites, the pictures of embryos stained by $n t l$ probe or $m y o D$ probe were measured by ImageJ software $1.47 \mathrm{v}$ (National Institutes of Health, USA) and analyzed as described previously $[6,41]$.

\section{Morpholinos, RNAs and microinjection}

Morpholinos (MOs, Gene Tools, LLC, USA) were designed to target the $5^{\prime}$ untranslated region (tb-MO) or the intron 3/ exon 4 boundary (sb-MO) of Drafp $4 a$ or Drafp $4 b$. Sequences were as following: afp $4 a$-tb-MO: 
5'-AAGAGTTGCCTTGCGTCCAGAGATT-3'; afp4asb-MO: 5'-CACTGTGCCATGAACAAACAAAAAC3'; afp 4b-tb-MO: 5'-ATGATTGTGGGATGAGCCAGG GTTG-3'; afp 4b-sb-MO: 5'-AAGCACTGCATCACAA AGACAATAC-3'. In addition, several control MOs, such as standard control MO (Ctrl-MO, 5'-CСТCCTACCTCAGTTACAATTTATA-3'), afp $4 a-$ sb-miMO (afp $4 a$-sb-MO with five-nucleotide mismatches indicated in lowercase, 5'-CACTcTcCCATG AAgAAtCtAAAAC-3'), and afp $4 b$-tb-miMO (afp $4 b$-tbMO with five-nucleotide mismatches indicated in lowercase, 5'-ATcATTGTcGGATcAGCCAcGcTTG-3') were also synthesized.

For rescue experiments, $a f p 4 a$ and $a f p 4 b$ cDNAs were amplified with primers containing BamHI and XhoI restriction sites from full-length cDNA without $5^{\prime}$ untranslated region (UTR) and cloned into pCS2+. To test the efficiency and specificity of tb-MOs, $5^{\prime}$ UTR and part of the $\mathrm{N}$-terminal open reading frame (ORF) of $a f p 4 a$ or $a f p 4 b$ were fused in frame with the EGFP ORF, and cloned into pCS2+ (primers are listed in Table 1). Plasmid for in vitro transcription of Kaede was generous gift from Dr. Brian Ciruna. Capped RNAs were prepared with the mMESSAGE mMACHINE kit (Ambion, USA) as previously described [12].

MOs or mRNAs were injected at the one-cell stage. The amount of $\mathrm{MO}$ or mRNA injected for each embryo was as below: afp $4 a$-sb-MO or afp $4 a$-sb-miMO, $4 \mathrm{ng}$; $a f p 4 b$-tb-MO or afp $4 b$-tb-miMO, $2.5 \mathrm{ng}$; afp $4 a$-tb-MO or afp $4 b-\mathrm{sb}-\mathrm{MO}, 8 \mathrm{ng}$; afp $4-\mathrm{MOs}, 4 \mathrm{ng}$ afp $4 a$-sb-MO and 2.5 ng afp $4 b$-tb-MO; Ctrl-MO was at the same amount with afp4-MO using in the same experiment; to detect the translation blocking efficiency, about 100 pg afp $4 a$ :EGFP or afp $4 b$ :EGFP mRNA was injected into a random subset of afp4-MO-injected embryos (co-injection); for rescue experiment, about 150 pg afp $4 a$ or $a f p 4 b$ mRNA was co-injected with afp4-MO.

\section{Reagent treatments}

For nocodazole treatment, the dechorionated embryos were incubated in $1 \mu \mathrm{g} / \mathrm{mL}$ nocodazole (Sigma, USA), which was diluted in $1 \times$ Danieau buffer as previously described [6]. For pregnenolone treatment, the dechorionated embryos were incubated with $0.1,1,10$ or $20 \mu \mathrm{M}$ pregnenolone (Sigma, USA) from $1 \mathrm{k}$-cell stage until the late epiboly stage.

\section{Microtubule and F-actin staining}

Embryos with chorion removed were fixed in microtubule-stabilizing buffer (MSB) and the whole-mount microtubule staining with primary antibody mouse anti- $\beta$-tubulin (1:500 in blocking buffer, Sigma, USA) and secondary antibody rhoda- mine-conjugated goat anti-mouse IgG (1:200 in blocking buffer, Thermo, USA) was performed according to previous study [42].

Images were acquired with a Leica TCS-SP2 confocal microscope (Leica, Germany), and confocal Z-series image stacks collected at $2 \mu \mathrm{m}$ intervals were assembled by LSM510 basic software. Microtubule area percentage (\%) was calculated as following steps. Firstly, several $100 \times 100$ pixel (about $72.2 \times 72.2 \mu \mathrm{m}$ ) images of the YCL microtubules were randomly intercepted from the blastoderm margin of each embryo picture, as indicated by a white square in the corresponding picture. Then, these images were changed to gray-scale images by Adobe Photoshop CS (Adobe, USA). Moreover, the gray-scale images were converted to white (microtubules) and black (background) by Make Binary of ImageJ. Finally, the pixel number of white (n) in each image was measured by Histogram of ImageJ, and the proportion in the $100 \mathrm{x}$ 100 pixel (n/10000) was computed as microtubule area percentage $(\%)$ in each image. For this calculation, more than 20 embryos were used, and about five images for each embryo were computed.

F-actin staining was carried out with $1 \mu \mathrm{g} / \mathrm{mL}$ rhodamine-phalloidin (Sigma, USA) as previously described [43]. Rhodamine-phalloidin was prepared as stock solutions of $1 \mathrm{mg} / \mathrm{mL}$ in methanol, and then diluted in PBTD/BSA just before use. Images were acquired with a Leica TCS-SP2 confocal microscope and confocal Z-series image stacks collected at $2 \mu \mathrm{m}$ intervals were assembled by LSM510 basic software. Phalloidin signal intensity, which indicated F-actin intensity, was analyzed using Image software as previously described [44].

\section{Cell lineage tracing}

Cell lineage tracing was performed according to previous reports [41, 45]. Briefly, 100 pg Kaede mRNA was injected with $a f p 4 b$-tb-miMO or afp $4 b$-tb-MO to embryos at the one-cell stage. The injected embryos were kept in the dark until the shield stage. The Kaede fluorophore was converted from green to red by focusing a 40-sec pulse of ultraviolet (UV) light, specifically on a group of cells in dorsal or lateral blastoderm margin, using the pinhole of a Leica LCS SP2 confocal microscope. Photos were acquired at the indicated developmental stages by a Leica MZ16 FA stereomicroscope (Leica, Germany). Figures were constructed using Adobe Photoshop CS. The angle and length were measured by utilizing ImageJ software.

\section{Statistical analyses}

For statistical analyses, means \pm standard deviation (SD) were acquired by Microsoft Excel 2003 (Mi- 
crosoft, USA), and one-way analyses of variance (ANOVA) and cross-table analyses were performed with SPSS 13.0 software (SPSS, USA).

\section{Results}

\section{Identification and molecular characterization of two tandem afp4s in gibel carp and zebrafish}

Previous report has characterized a full-length cDNA of afp4 in gibel carp (GenBank accession No. AY365004) [20]. To characterize its genomic organization, we obtained a positive BAC by PCR screening from gibel carp BAC library [32] as described previously [10]. Sequencing the BAC clone revealed the two tandem duplicated gene sequences, and full-length cDNA of the other afp4 was achieved by RACE (GenBank accession No. KJ183062). Zebrafish database searches also found a similar genomic organization of the two tandem duplicated genes on the chromosome 16 (Supplementary material Fig. S1), and revealed two afp 4 homologues (GenBank accession No. BC133822 and BC153962). Then, full-length cDNAs of the two afp4s was reexamined and acquired by RACE (GenBank accession No. KJ183061 and BC153962). Genomic organization comparison between gibel carp and zebrafish revealed almost identical exons in size and similar exon/intron boundaries (Supplementary material Table S1).

In order to clarify evolutionary relationship between the four AFPs and other functionally characterized AFP4s with antifreeze activity, we constructed a phylogenetic tree. As shown in Fig. 1A, although the classification position of these species belongs to different orders including Ostariophysi, Paracanthopterygii and Acanthopterygii, the eight known AFP4s are gathered into two main clades. Clade A contains DrAFP4s and CagAFP4s, and clade B includes the four functionally characterized AFP4s. Multiple amino acid sequence alignment of DrAFP4s and other AFP4s also revealed their evolutionary conservation. Significantly, the newly characterized AFPs in zebrafish and gibel carp shared common properties of the functionally characterized AFP4s, including the four a-helical regions, signal peptide, unrelated coding region 1 and 2 (UCR1 and UCR2), the 33-codon block, and 11-mer repeats [23-26]. Moreover, the amino acid identities between DrAFP4a and other AFP4 ranged from 31.1\% to $83.8 \%$, and their signal peptide and a-helical region showed higher consensus than the complete amino acid sequences (Fig. 1B). Therefore, the newly identified and characterized genes should belong to homologs of afp4. Drafp $4 a$ and Drafp $4 b$ were abbreviated to $a f p 4 a$ and $a f p 4 b$ for the common use.
To further investigate the biological functions of afp $4 \mathrm{~s}$ in zebrafish, we analyzed the full-length cDNA sequences of zebrafish $a f p 4 a$ and $a f p 4 b$ (GenBank accession No. KJ183061 and BC153962, respectively). As shown in Fig. 1C, their nucleotide sequence alignment shows $88.8 \%$ of high identity in the ORFs, but much lower identity exists in the $5^{\prime}$ and $3^{\prime}$ UTR regions, and the 3' UTR of $a f p 4 b$ is $275 \mathrm{nt}$ longer than that of $a f p 4 a$. Moreover, significant sequence differences were found in their predicted promoter regions in $5^{\prime}$ upstream sequences (data not shown). All the sequence differences imply the existence of their divergence in expression pattern and biological function.

\section{Divergent and dynamic temporal expression patterns between afp $4 a$ and $a f p 4 b$}

Subsequently, we examined expression patterns of $a f p 4 a$ and $a f p 4 b$ in zebrafish by qPCR and Western blot analyses. Firstly, two sets of specific primers were designed according to their most divergent sequences to detect and distinguish afp $4 a$ transcript and afp $4 b$ transcript, respectively (Fig. 1C), and the transcript specificity was verified by sequencing the amplified products of afp $4 a$ and $a f p 4 b$. Significantly, the qPCR revealed differential expression pattern and dynamic changes between $a f p 4 a$ and $a f p 4 b$ during embryogenesis and early larval development. As shown in Fig. 2A, afp $4 a$ initially transcribes from 4 hours postfertilization (hpf) at which the embryos develop to sphere stage, whereas $a f p 4 b$ transcribes from $6 \mathrm{hpf}$ when the embryos are at shield stage. As development proceeds, afp $4 a$ transcript increases progressively and reaches to the highest level at $12 \mathrm{hpf}$ when the embryos develop to 6-somite stage, whereas the amount of $a f p 4 b$ transcript exceeds that of $a f p 4 a$ at $12 \mathrm{hpf}$, and peaks at $16 \mathrm{hpf}$. At the $a f p 4 b$ peak duration, afp $4 a$ transcript gradually decreases from $12 \mathrm{hpf}$ to $20 \mathrm{hpf}$. Then, when the $a f p 4 b$ transcript reduces gradually from $20 \mathrm{hpf}$ to $28 \mathrm{hpf}$, afp $4 a$ transcript rises again from $24 \mathrm{hpf}$ to $28 \mathrm{hpf}$. Around $32 \mathrm{hpf}, a f p 4 b$ increases again, and quickly declines to the bottom from $32 \mathrm{hpf}$ to 48 hpf. After $48 \mathrm{hpf}$, almost no afp $4 b$ transcript is detected in the later embryos and larvae. However, the afp $4 a$ transcript decreases again from $32 \mathrm{hpf}$ to $72 \mathrm{hpf}$, but its transcript gains once again in $96 \mathrm{hpf}$ and $120 \mathrm{hpf}$ larvae. The AFP4s protein expression pattern during embryogenesis was also detected by Western blot assay using the antibody against both AFP4s. As shown in Fig. 2B, the specific AFP4s protein band appears from $6 \mathrm{hpf}$ at shield stage, reaches to the peak level at $16 \mathrm{hpf}$, and then reduces gradually to a certain level from $24 \mathrm{hpf}$ to $96 \mathrm{hpf}$ during later embryogenesis. 
A

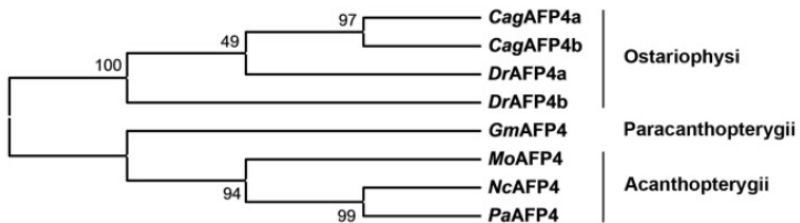

B

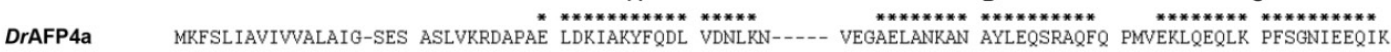
DrAFP4b MRLSLITILVVALAIR-SES ASLVKRDASAE LDKIAKYFQDF VDNLKN----- VEGPELANKAN AYLEESRAQFQ PMVEKLQEQLK PFS-NIEDQIK CagAFP4a MRFFLIAVLVTALAIG-SES VSLVKRDAPAE LEKITKYFQDL VDNLKH----- VEGPELVNKAN AYFEQSRAQFQ PMVERLQEQLK PLSSNIEDHIK CagAFP4b MRFSLITILVVALAIG-SES VSLVRRDAPAE LEKIAKYFQDL VDNLKH----- VEGPELVNKAN AYFEQSRAQFQ PMVERLQEQLK PLSSNIEDHIK NCAFP4 MRFSLIAAVVLLALAQGSFA ----QD-SAD LERLGRYLEDM KDKMVRELTDI INTHDLANQAT SFVNEKKIQLE PLVAQIQEQLQ AVASNAEAQIK PaAFP4 MKFSLIAAVVLLALAQGSFA ----QD-AAD LERLGQYLEDM KNKMVLDLTEI IRTHDLANQAT TFVNEKKTQLE PLVAHIQEQLQ TVASNAEAQIK MoAFP4 MRFSLVATIVLLALAQGSFA ----QG-AAD LESLGQYFEEM KTKLIQDMTEI IRSQDLANQAQ AFVEDKKTQLQ PLVAQIQEQMK TVATNVEEQIR GmAFP4 MKYTLIAAIVVLALAQGTLA -----VEQSPE LEKMAQRFEGM KTELMATVQRV SES----LQSQ TIIEDGRTQLE PIMTQIQEHLA PLATSVQEKVT

CD linker D

DrAFP4a

DrAFP4b

CagAFP4a

CagAFP4b

NcAFP4

PaAFP4

MOAFP4

GmAFP4

Consensus PLAASVQSQVA PLAGMVQTHVE DMIKFVADQARAMLPPQ PIAASVQAQVA PLADIVQTHIE DVLKFVADKTKTILPPQ PLAASVQAQVA PLASMIQTHVE DVLKFVADTSKAILPQPLAASVQAQVA PLASMIQTHVE DVLKFVADKSKAILPPQ PLAANVQAQFQ PQIDSFQQQID AIMQQLTRPAAPIAN-PLAANVQAQFQ PQIDSFQQQME AIFQQLTRPAAPIDN-PLTANVQAHLQ PQIDNFQRQME AIIKKLTDQTMAIEN-PLAEDMQQRLK PYVDEFQSELE SVLRKLLDQAKAITQ-$*:: . *: . \quad * . . * \ldots:$ : : : : : : : .: $|\ldots \ldots 7 \ldots||\ldots 8 \ldots \ldots||\ldots \ldots 9 \ldots| \mid$.UCR2 $\mid$

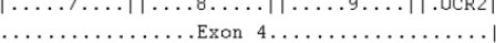

$\begin{array}{lcc} & \text { Identity (\%) } & \text { Similarity (\%) } \\ 130 & & \\ 129 & 83.1 & 92.3 \\ 129 & 81.5 & 89.2 \\ 130 & 83.8 & 93.1 \\ 128 & 33.8 & 59.6 \\ 128 & 35.3 & 58.1 \\ 128 & 36.8 & 59.6 \\ 125 & 31.1 & 59.8\end{array}$

C

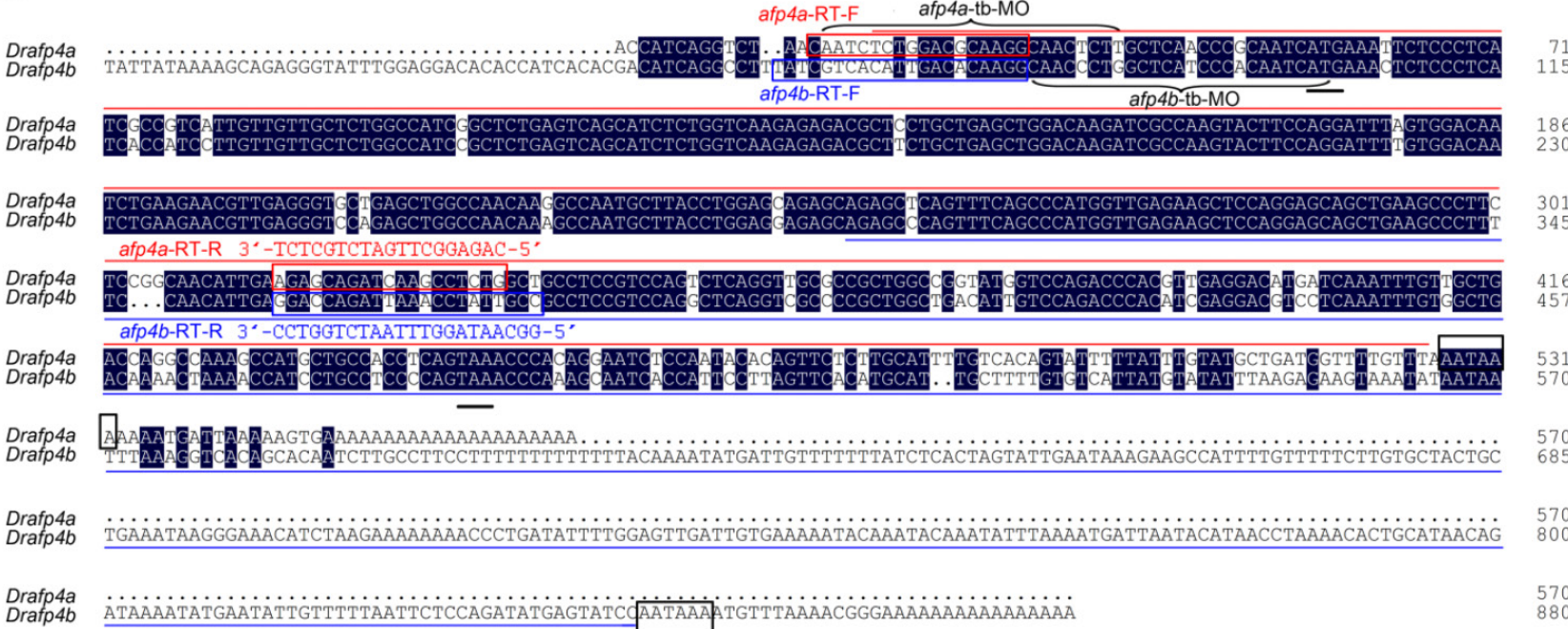

Figure I. Phylogenetic relationship and molecular characterization of DrAFP4s and other functionally characterized AFP4s. (A) An unrooted ML phylogenetic tree of the known AFPs from Danio rerio (DrAFP4a, NP_001038953; DrAFP4b, XP_69709I), Carassius auratus gibelio (CagAFP4a, AHZ08737; CagAFP4b, AARI299I), Gadus morhua (GmAFP4, Q56TU0), Myoxocephalus octodecemspinosus (MoAFP4, ABA4I379), Notothenia coriiceps (NcAFP4, ADU02I8I) and Pleuragramma antarcticum (PaAFP4, ADU02I80). Their classification positions, such as Ostariophysi, Paracanthopterygii and Acanthopterygii, are indicated on the right. (B) Multiple amino acid sequence alignment of DrAFP4s and other AFP4s. Amino acids consensus is shown below the alignment by three kinds of consensus symbols. An asterisk $(*)$ means a position where has a single conserved residue; a colon (:) means a position where process high similarity (scoring $>0.5$ ); a period (.) means a position where hold low similarity (scoring $=<0.5$ ). Asterisks $(*)$ above protein sequences show the predicate $\alpha$-helical regions. Three exons (E2, E3 and E4) and boundary of eight parts of proteins [signal peptide, unrelated coding region I and 2 (UCR I and UCR2), the 33-codon block, and I I-mer repeats (4 to 9)] are indicated according to a previous report [25]. Identities and similarities between DrAFP4a with other AFP4s are shown behind the alignment. (C) Nucleotide sequence alignment of Drafp $4 a$ and Drafp4b cDNAs. Identical nucleotides are indicated by the black background; the start code (ATG) and stop code (TAA) are lined by black lines; putative polyadenylation signals are boxed; the targets of the antisense probe to afp $4 a$ or $a f p 4 b$ used in whole-mount in situ hybridization are lined by red line above or blue line below sequences, respectively; two sets of specific primers used in RT-PCR to detect afp $4 a$ or afp $4 b$ transcript are respectively indicated by red or blue boxes; targets of tb-MOs are pointed out by brackets. 
A

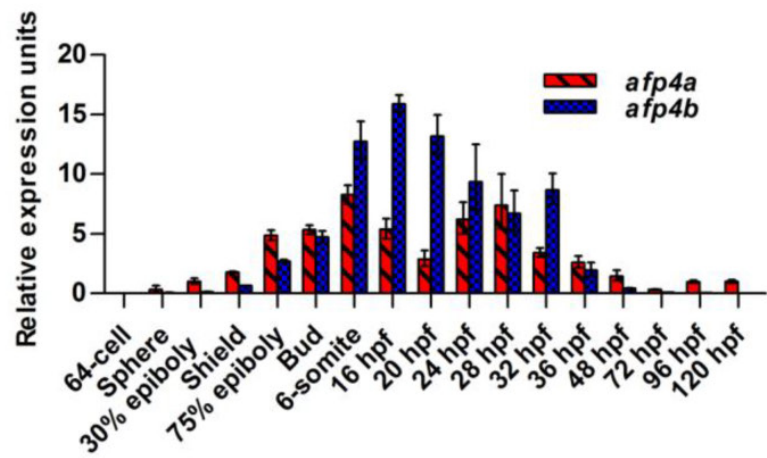

B

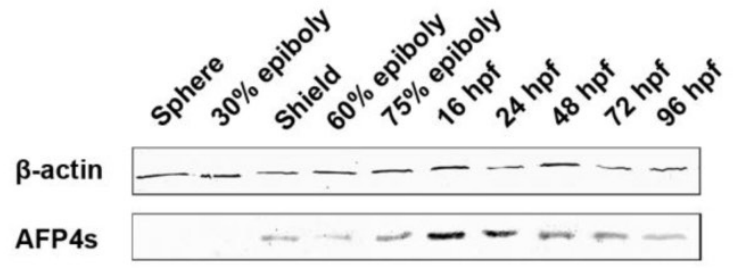

\section{C afp4alafp4b}

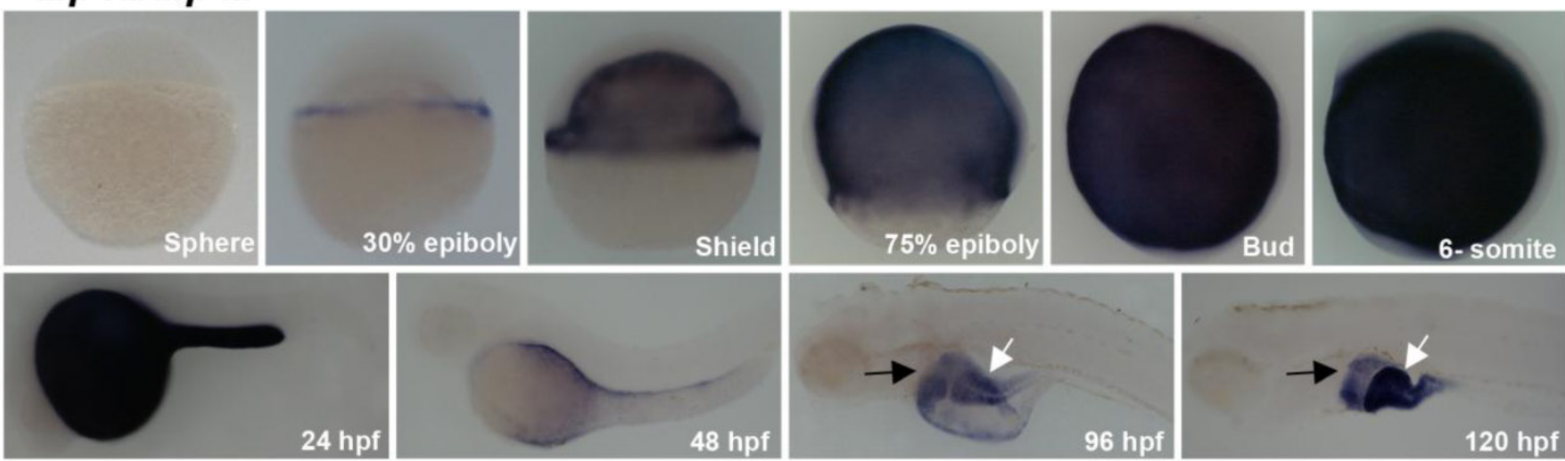

\section{D afp4b}
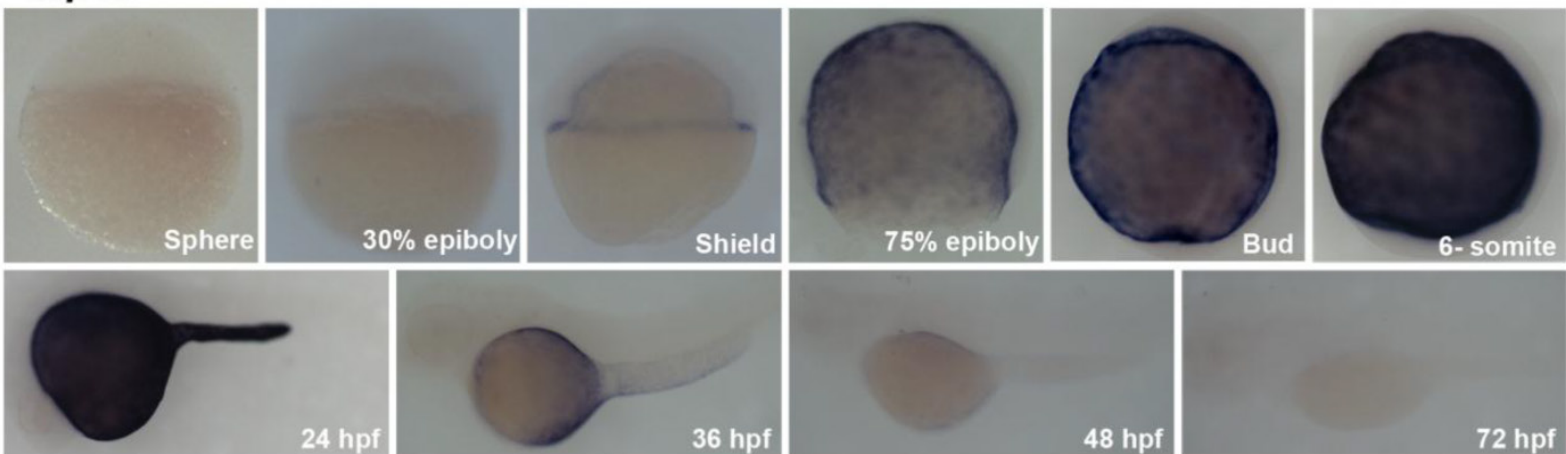

E

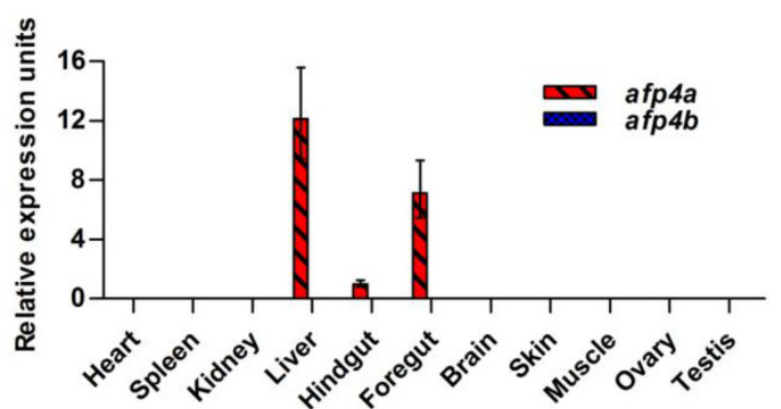

$\mathbf{F}$

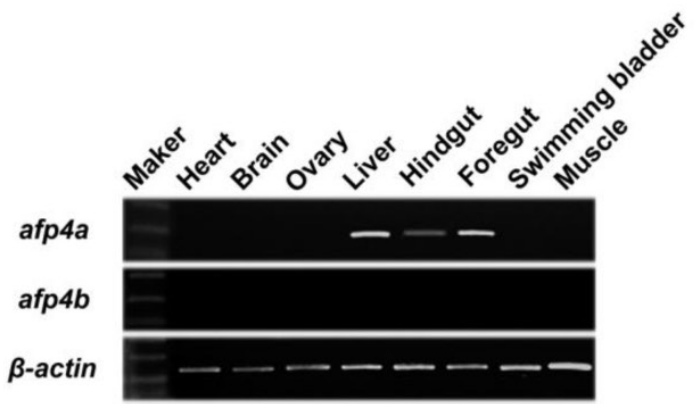

Figure 2. Temporal and spatial expression pattern of $a \mathrm{fp} 4 \mathrm{~s}$. (A) Expression of $a \mathrm{fp} 4 a$ and $a \mathrm{fp} 4 \mathrm{~b}$ in embryonic development stages and early larvae evaluated by $\mathrm{qPCR}$ and normalized to $\beta$-actin. The histograms stand for the relative transcript level of afp $4 a$ (red) or afp $4 b$ (blue), and the relative transcript level of afp $4 a$ at the sphere stage is set to I. Data shown are means \pm SD of three independent assays. (B) Western blot assay from sphere stage to 96 hpf. $\beta$-actin protein is internal control. (C, D) In situ hybridized WT embryos for $a f p 4 a / a f p 4 b(C)$ or $a f p 4 b$ (D). Stages of embryos are shown in the bottom right corner of each panel. Black arrows indicate the liver, and white arrows indicate the intestine. Embryos from sphere stage to 6-somite stage are oriented animal pole up and dorsal right, and embryos from $24 \mathrm{hpf}$ to $120 \mathrm{hpf}$ are heading to the left and dorsal up. $(E, F)$ Expression of afp $4 a$ and afp $4 b$ in adult tissues evaluated by $q P C R(E)$ and semi-quantitative RT-PCR (F), and normalized to $\beta$-actin. (E) The histograms stand for the relative transcript level of $a f p 4 a$ (red) or afp $4 b$ (blue), and the relative transcript level of $a f p 4 a$ in the hindgut is set to I. Data shown are means \pm SD of three independent assays. 


\section{Yolk syncytial layer and digestive system-specific expression of afp4s in embryos, early larvae, and adults}

Moreover, the expression patterns of afp $4 a$ and $a f p 4 b$ were investigated in embryos and larvae by WISH using two different antisense probes. The first probe, targeted to a 5'-terminal $506 \mathrm{nt}$ sequence of afp $4 a$ transcript (Fig. 1C), can recognize both transcripts of $a f p 4 a$ and $a f p 4 b$ because of their high sequence similarity. The second probe, which is mainly against to extra 3'-terminal of afp $4 b$ with 553 nt (Fig. $1 C)$, is only able to examine the $a f p 4 b$ transcript. These WISH data further revealed a differential and dynamic expression fashion between $a f p 4 a$ and $a f p 4 b$. As shown in Fig. 2C, the positive signal hybridized with the first probe initiates to appear in YSL from 30\% epiboly stage at about $4.7 \mathrm{hpf}$. Then, the signal quickly enhances from shield stage at about $6 \mathrm{hpf}$, and reaches to the strongest from $8 \mathrm{hpf}$ to $12 \mathrm{hpf}$ during which $75 \%$ epiboly, tail-bud formation and somitogenesis (6-somite) have progressed. The sectioned observations of the stained embryos from shield to bud stages showed restricted expression in YSL (Supplementary material Fig. S2A-C). Along with the embryonic development, the specific expression domain in YSL becomes clearer, especially in $24 \mathrm{hpf}$ embryos. After yolk absorption, the signal gradually becomes weak from the posterior to anterior YSL in the hatched larvae, and finally restricts to the formed digestive organs, including intestine and liver, in $96 \mathrm{hpf}$ and 120 hpf larvae. In comparison with the above data, the $a f p 4 b$-specific transcript expresses later, and also appears within YSL from the shield stage embryos at about $6 \mathrm{hpf}$. Then, its expression strengthens rapidly, and reaches the strongest at about $12 \mathrm{hpf}$ when the embryos develop to 6-somite stage (Fig. 2D). The longitudinal section of the stained embryos also showed specific expression in YSL (Supplementary material Fig. S2D-F). After $24 \mathrm{hpf}$, the $a f p 4 b$-specific transcript reduces gradually, and not any afp $4 b$-specific transcript is observed in the corresponding expression position after $72 \mathrm{hpf}$ (Fig. 2D).

Furthermore, tissue distribution of $a f p 4 a$ and $a f p 4 b$ transcripts was examined in adult zebrafish by qPCR and semi-quantitative RT-PCR. As shown in Fig. $2 \mathrm{E}$ and $2 \mathrm{~F}$, the afp $4 a$ transcript is abundant in liver and foregut, slight in hindgut, and no signal exists in other tissues, including heart, spleen, kidney, brain, skin, muscle, ovary, testis and swimming bladder, whereas afp $4 b$ mRNA is absent in all the examined tissues. All the data indicate that both transcripts of $a f p 4 a$ and $a f p 4 b$ express specifically in YSL, but the later expressed $a f p 4 b$ appears only in embryogenesis, whereas afp $4 a$ expresses continually in YSL and di- gestive system from early embryos to adults, suggesting that $a f p 4 a$ and $a f p 4 b$ might play similar but different biological roles during zebrafish embryogenesis and early larval development.

\section{AFP4a and AFP4b contribute to early morphogenetic movements during zebrafish embryogenesis}

To explore their respective biological functions, we designed two kinds of antisense MOs specific to $a f p 4 a$ or $a f p 4 b$. One is translation-blocking $\mathrm{MO}$ (tb-MO), which is directed to the 5' UTR of afp $4 a$ (afp $4 a$-tb-MO) or $a f p 4 b$ (afp $4 b$-tb-MO) (Fig. 1C). The efficiency and specificity of each tb-MO were confirmed by co-injecting with afp $4 a$ :EGFP or afp $4 b: E G F P$ mRNA (100 pg/embryo) into one-cell stage zebrafish embryos. The test showed that about $8 \mathrm{ng}$ afp $4 a$-tb-MO for each embryo could largely deplete the EGFP fluorescence produced by afp 4a:EGFP mRNA, but did not inhibit that expressed by $a f p 4 b$ :EGFP mRNA. On the other hand, lower dose afp $4 b$-tb-MO (2.5 ng/embryo) could completely deplete the EGFP fluorescence produced by afp $4 b$ :EGFP mRNA, but did not reduce that produced by afp4a:EGFP mRNA. However, if the dose of $a f p 4 b$-tb-MO was increased to $5 \mathrm{ng}$, the proportion of afp 4a:EGFP-injected embryos with EGFP fluorescence was significantly reduced to 62\% (Supplementary material Fig. S3A). These data indicate that afp $4 a$-tb-MO can specifically knockdown AFP4a expression, whereas $a f p 4 b$-tb-MO can specifically deplete AFP4b expression only at a lower dose, and a higher dose can interfere with the specificity and lead to cross-reaction with afp $4 a$.

The other kind of MO is splice-blocking morpholino (sb-MO), which is targeted to the intron 3 /exon 4 boundary of afp $4 a$ (afp $4 a$-sb-MO) or afp $4 b$ (afp $4 b$-sb-MO), since significant nucleotide difference only exists in the boundary sequence between afp $4 a$ and $a f p 4 b$ premature mRNAs (Supplementary material Table S2). The sb-MO resulted in a transcript with intron 3 insertion, and introduced a premature stop codon right after the exon $3 /$ intron 3 junction. This early stop codon led to a truncated protein (58 amino acid residues), which lost the entire product of exon 4, including the characteristic 11-mer conserved repeats in all AFP4s (Fig. 1B, Supplementary material Fig. S3B and Table S2). Semi-quantitative RT-PCR results showed that $4 \mathrm{ng}$ afp $4 a$-sb-MO could specifically change the splice of all the endogenous afp $4 a$ mRNA, while $8 \mathrm{ng} a f p 4 b$-sb-MO could only specifically change the splice of about half the endogenous afp $4 b$ mRNA (Supplementary material Fig. S3B).

Then, 4 MOs were respectively injected with appropriate concentration described above. When 
Ctrl-MO (8 ng/embryo) embryos developed to bud stage, both afp $4 a$-tb-MO ( $8 \mathrm{ng} /$ embryo) morphants and afp $4 a$-sb-MO (4 ng/embryo) morphants displayed an epibolic delay; nevertheless, a more serious defect in epiboly was observed in afp $4 a$-sb-MO morphants. At the same time, both afp $4 b$-tb-MO (2.5 ng/embryo) morphants and afp $4 b$-sb-MO (8 ng/embryo) morphants showed a longer anterior-posterior axis, and $a f p 4 b$-sb-MO led to a similar but milder defect (Supplementary material Fig. S3C). The two distinct MOs targeted to the same gene caused the similar defect, confirming the phenotypes-specific to $a f p 4 a$ or $a f p 4 b$. Moreover, afp $4 a$-sb-MO and $a f p 4 b$-tb-MO could produce the more obvious defect with lower individual MO dose than the other MO targeted to the same gene, and the lower dose was likely to cause fewer non-specific defects. Therefore, afp $4 a$-sb-MO and afp $4 b$-tb-MO were used in subsequent experiments.

Subsequently, afp $4 a$-sb-MO (4 ng/embryo) and afp $4 b$-tb-MO (2.5 ng/embryo) were respectively or simultaneously (afp4-MOs) injected into zebrafish embryos at the one-cell stage. Western blot detection further confirmed the specificity and effectiveness. A significant decrease of AFP4s level was observed from shield stage (about $92 \%$ reduction) to 6-somite stage (about $64 \%$ reduction) in the afp $4 a$-sb-MO morphants, whereas a contrary change was detected from shield stage (about $6 \%$ reduction) to 6 -somite stage (about $38 \%$ reduction) in the $a f p 4 b$-tb-MO morphants (Fig. 3A and $3 \mathrm{~B})$. These are consistent with the higher transcript level of $a f p 4 a$ than that of $a f p 4 b$ before 6 -somite stage. Significantly, all the AFP4s were completely depleted from shield stage to 6-somite stage in the afp $4 a$-sb-MO and afp $4 b$-tb-MO co-injected (afp4-MOs) morphants.

Moreover, morphologic changes of embryos in each group were closely monitored. When Ctrl-MO embryos developed to shield stage, at which time their blastoderm covered $50 \%$ of the yolk and formed shield, afp 4a-sb-MO morphants also formed shield, but their blastoderm could not cover half of the yolk. While Ctrl-MO embryos reached 75\% epiboly, afp $4 a$-sb-MO morphants displayed an obvious delay in epiboly. At the bud stage, when epiboly was completed in Ctrl-MO embryos, about $20 \%$ yolk could not be covered by blastomeres in afp $4 a$-sb-MO morphants (Fig. 3C, D). Significantly, the afp $4 b$-tb-MO injected embryos did not show any obvious defects until 75\% epiboly, but the morphants began to display a longer anterior-posterior axis from bud stage. Moreover, delayed developments were observed during somitogenesis, in which their somites became wider in the mediolateral axis and the boundary of somites was faint. At $24 \mathrm{hpf}$, the $a f p 4 b$-tb-MO injected embryos displayed shorter anterior-posterior body axis without distinct $\mathrm{V}$-shape somites, and approximately $49.3 \%$ of the morphants died (Fig. 3E, G). In contrast, the afp $4 a$-sb-MO injected embryos showed a more normal phenotype than the afp $4 b$-tb-MO morphants after bud stage. At 6 -somite stage, about $85.4 \%$ morphants, which had closed their blastopores, showed a slight increased linear distance between the head and tail, and about $8.0 \%$ morphants still could not finish epiboly (data not shown). However, some obvious defects, such as curving trunk, reduction of yolk extension and diminution of head growth, were observed at $24 \mathrm{hpf}$. Meanwhile, the $8.0 \%$ afp $4 a$-sb-MO morphants, which failed to finish epiboly, displayed a curly tail (Fig. 3D). In comparison with the afp $4 a$-sb-MO (Fig. 3D) or afp $4 b$-tb-MO (Fig.3E) morphants, the morphogenetic defects during gastrulation (from shield stage to bud stage) in the afp4-MOs co-injected morphants (Fig. 3F) were basically similar to that in the embryos merely injected with afp $4 a$-sb-MO (Fig. 3D). However, the proportion of the dead afp4-MOs embryos was higher (about 33.3\%) than that of the embryos only injected with afp $4 a$-sb-MO (about $2.4 \%$ ) or afp $4 b$-tb-MO (about $2.5 \%$ ) at bud stage (Fig. 3G). Two hours later, when embryos developed to 6-somite stage, the survived afp4-MOs morphants showed the delayed development of head, elongated anterior-posterior axis and faint somite boundary, or even crooked notochord. Furthermore, the proportion of dead afp4-MOs-injected embryos increased substantially to approximately $83.1 \%$, whereas those of single MO injected embryos were still very low (about 3.5\% for afp $4 a$-sb-MO, and 3.9\% for $a f p 4 b$-tb-MO). At $24 \mathrm{hpf}$, the survived afp $4-\mathrm{MOs}$ morphants showed similar phenotypes with afp $4 b$-tb-MO morphants, and the proportion of dead embryos increased to about $86.8 \%$. (Fig. 3F, G)

To confirm the specificity for above data, we synthesized mRNA of $a f p 4 a$ and $a f p 4 b$ lacking 5' UTR, where was the target of tb-MO for rescue examination. Remarkably, the morphogenetic defects caused by the afp4-MOs could be rescued by the synthesized mRNA of $a f p 4 a$ and $a f p 4 b$, because significant death decrease and significant wild type increase appeared in the co-injected embryos of each or mixture of afp $4 a$-mRNA and afp $4 b$-mRNA (P < 0.001). However, phenotype and death were still at high levels. (Fig. 3G) This observation could because that the RNA amount injected to embryos was not most suitable for rescue. These data suggest that AFP4a and AFP4b might contribute to early morphogenetic movements during zebrafish embryogenesis. 
A

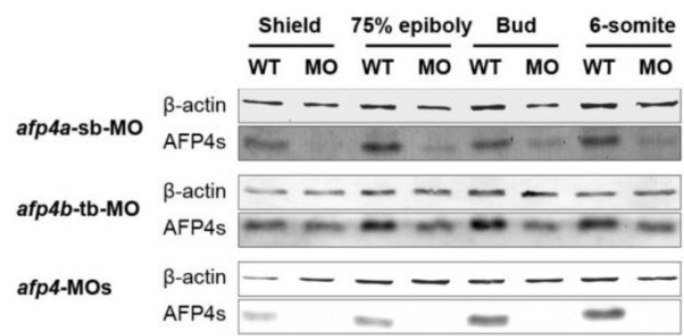

B

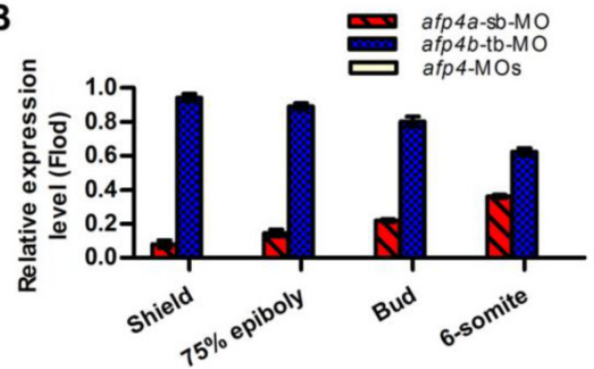

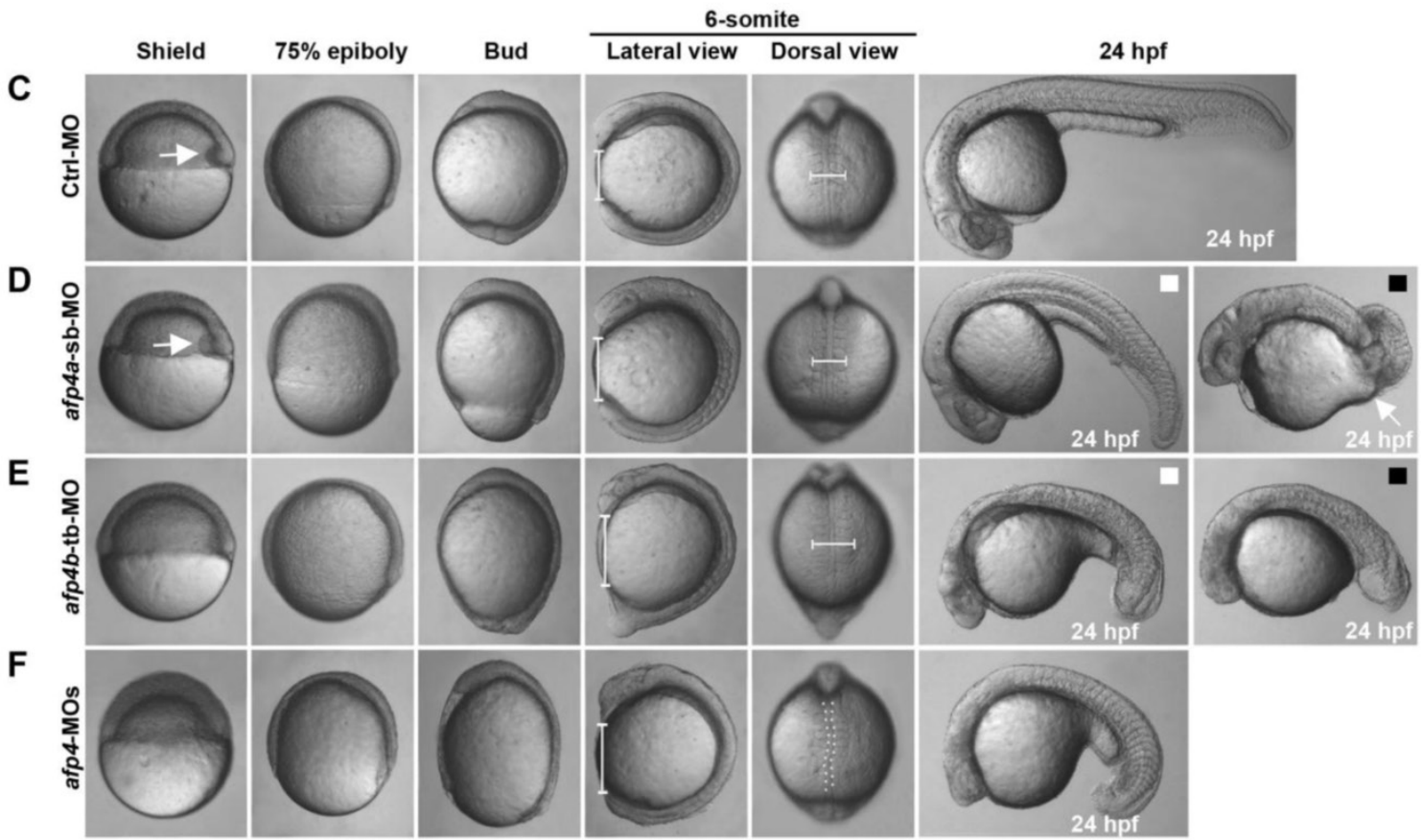

G

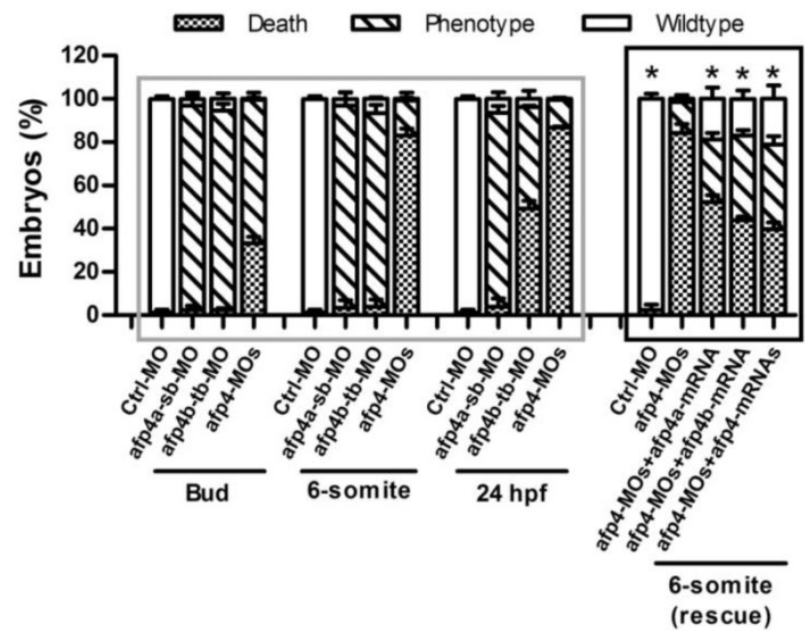

Figure 3. Reduced AFP4a and/or AFP4b expression causes serious morphogenetic defects during early embryonic development. (A) Test of decrease of AFP4s by Western blotting at shield stage, 75\% epiboly stage, bud stage and 6-somite stage after injection of afp4a-sb-MO and/or afp4b-tb-MO. afp4-MOs means afp4a-sb-MO and afp4b-tb-MO co-injection. $\beta$-actin protein is internal control. (B) Statistical data of the relative AFP4s expression level in afp $4 a$-sb-MO and/or afp $4 b$-tb-MO morphants from shield stage to 6 -somite stage. Normalized to the expression of $\beta$-actin protein and the corresponding relative expression level of AFP4s in WT embryos is set to I. Results are presented as means \pm SD of three independent experiments. (C-F) Morphology of embryos from shield stage to 24 hpf after injecting Ctrl-MO (C), afp4a-sb-MO (D), afp4b-tb-MO (E), or afp4-MOs (F). Lateral views of embryos from shield to bud stage, dorsal to the right, anterior at the top; lateral or dorsal views of embryos at 6-somite stage with their animal pole facing upward; lateral views of embryos at $24 \mathrm{hpf}$, anterior towards the left and dorsal to the top. The arrows in shield stage embryos indicate the shield; the arrow in 24 hpf afp 4a-sb-MO morphants indicates the unclosed blastopore; the lines in lateral-viewed 6-somite stage embryos indicate head-to-tail distance; the lines in dorsal-viewed embryos indicate somites width; the dotted lines show the boundary of a curve notochord. Morphology was scored as mild (white) or severely defective (black) in afp4a-sb-MO or afp $4 b$-tb-MO injected embryos at 24 hpf. (G) Percentage of embryos at bud stage, 6-somite stage and 24 hpf in each class after injecting Ctrl-MO, afp $4 a$-sb-MO, afp $4 b$-tb-MO, or afp 4-MOs (the left columns indicate by a grey box. Results represent means \pm SD of three independent experiments, $N=76$ to II 6 embryos per condition); or percentage of afp4-MOs-injected embryos in each class at 6-somite stage after injecting afp4a-mRNA, afp4b-mRNA, or afp4a-mRNA together with afp4b-mRNA (afp4-mRNAs) (the right columns indicate by a black box. Results represent means \pm SD of three independent experiments, $N=55$ to 89 embryos per condition. $*$ means $P<0.00$, when compared to afp4-MOs). 
A

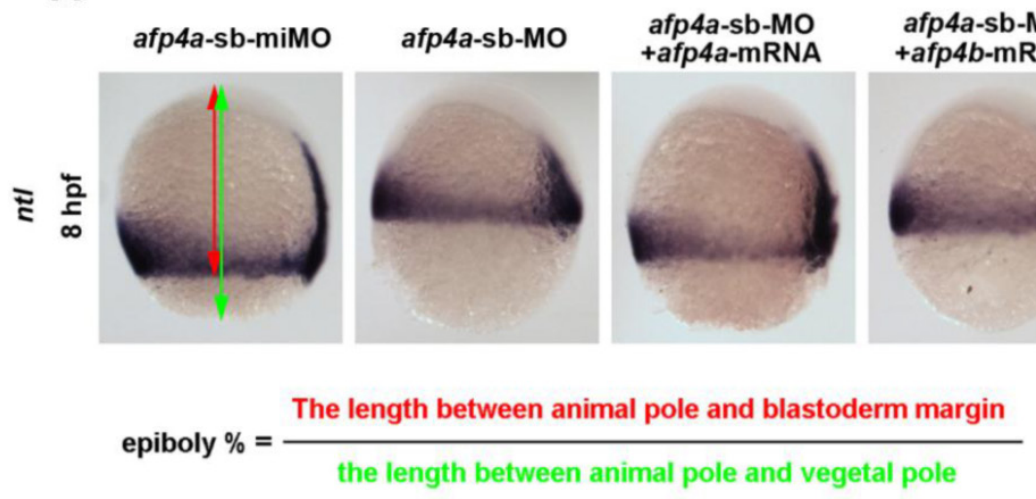

B

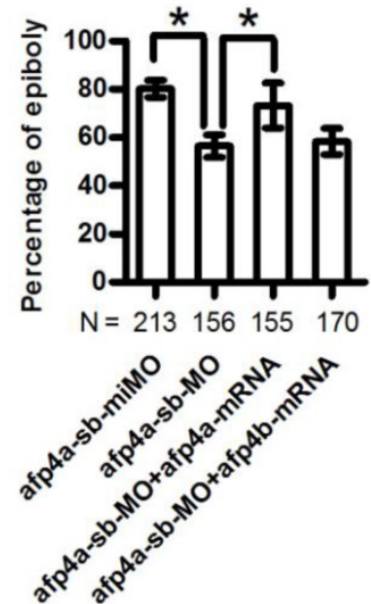

Figure 4. The reduced AFP4a expression leads to epibolic delay. (A) ntl expression in 8 hpf groups injected with afp4a-sb-miMO, afp4a-sb-MO, afp4a-sb-MO plus afp4a-mRNA, or afp 4a-sb-MO plus afp 4b-mRNA. Lateral views of embryos, dorsal to the right, anterior to the top. The calculating method for the epiboly percentage is shown. (B) Histogram represents mean $\pm \mathrm{SD}$ of the epiboly percentage in each group. $\mathrm{N}$, the number of embryos that were analyzed from three independent experiments. $* \mathrm{P}<0.0 \mathrm{I}$.

\section{AFP4a is required for epiboly progression}

To confirm whether the morphogenetic defect in the afp $4 a$-sb-MO morphants during gastrulation was resulted from AFP4a depletion, we further synthesized a control $\mathrm{MO}$ containing 5-base mismatches relative to afp $4 a$-sb-MO (afp $4 a$-sb-miMO). Identically to the above Ctrl-MO, the embryos injected with $4 \mathrm{ng}$ of afp $4 a$-sb-miMO were normal, and thus the afp $4 a$-sb-miMO was utilized as a control in following studies on the role of AFP4a. In addition, the synthesized $a f p 4 a$ or $a f p 4 b$ mRNA was co-injected with afp $4 a$-sb-MO for rescue examination, and the mesodermal marker $n t l$ was used to visualize the blastoderm margin through WISH (Fig. 4A) and thereby to quantify the epiboly percentage (Fig. 4B). In comparison with normal epiboly progression $(80.2 \% \pm 3.7 \%$, $\mathrm{N}=213$ ) at $8 \mathrm{hpf}$ in the afp $4 a$-sb-miMO embryos, the afp $4 a$-sb-MO embryos displayed significant epiboly delay $(56.5 \% \pm 5.0 \%, \mathrm{~N}=156)$, and the delay could be rescued by co-injection with 150 pg afp $4 a-m R N A$ $(73.1 \% \pm 9.3 \%, \mathrm{~N}=155)$, but could not be restored by the same amount of afp $4 b$-mRNA $(58.3 \% \pm 6.1 \%$, $\mathrm{N}=170$ ). These data indicate that AFP4a depletion leads to epiboly defect.

Since the afp $4 a$-expressed YSL had been revealed to play significant roles in germ layer differentiation and early morphogenetic movements [46], we further tested whether the AFP4a depletion affected other early embryonic development except for epiboly progression. Firstly, we detected germ layer differentiation by endodermal marker fox $A 3$, mesendodermal marker gsc and mesodermal marker $n t l$ in shield stage embryos. In comparison with the afp $4 a$-sb-miMO control embryos, their expression domains at the gastrulation onset stage were only located slightly towards animal-pole in the afp $4 a$-sb-MO morphants (Supplementary material Fig. S4A-C). These changes should be caused by epiboly delay, and germ layer differentiation is little affected in the AFP4a-knockdown embryos. At the same stage, AFP4a-depleted embryos also showed the prominent embryonic shield similar to that in control embryos, even though the blastoderms in these embryos covered less than $50 \%$ of the yolk cell (Fig. 3D). This phenotype implies that involution movement is normal in the morphants.

Moreover, localization of $n t l$ in axial chordal mesoderm and myoD in adaxial cells at bud stage confirmed the presence of a slight wider and shorter axis with the unclosed blastopore in afp $4 a$-sb-MO morphants (Supplementary material Fig. S4D and E), but the expression domains of myoD in adaxial cells and somites were comparable between afp $4 a-\mathrm{sb}-\mathrm{MO}$ morphants and afp $4 a$-sb-miMO morphants at 8-somite stage when most afp $4 a$-sb-MO morphants finished epiboly (Supplementary material Fig. S4F). These data indicate that mediolateral convergence seems a little change because of the unclosed blastopore. On the other hand, the normality in anterior part of $n t l$ expression domain was observed (Supplementary material Fig. S4G). Moreover, the expression of hgg1, which marked the prechordal plate, and of $d l x 3 b$, which expressed in the anterior edge of the neural plate, were almost the same as afp $4 a$-sb-miMO embryos at the bud stage (Supplementary material Fig. $\mathrm{S} 4 \mathrm{H})$. In addition, at the 8-somite stage, the expression domains of pax2.1 in the optic stalk, the midbrain hindbrain boundary, and the otic vesicles were comparable between afp $4 a$-sb-MO morphants and afp 4a-sb-miMO morphants (Supplementary material Fig. S4I). These data indicate that the anterior extension of the axial mesendoderm is not affected, and the convergence movement is slightly affected by AFP4a deficiency. 


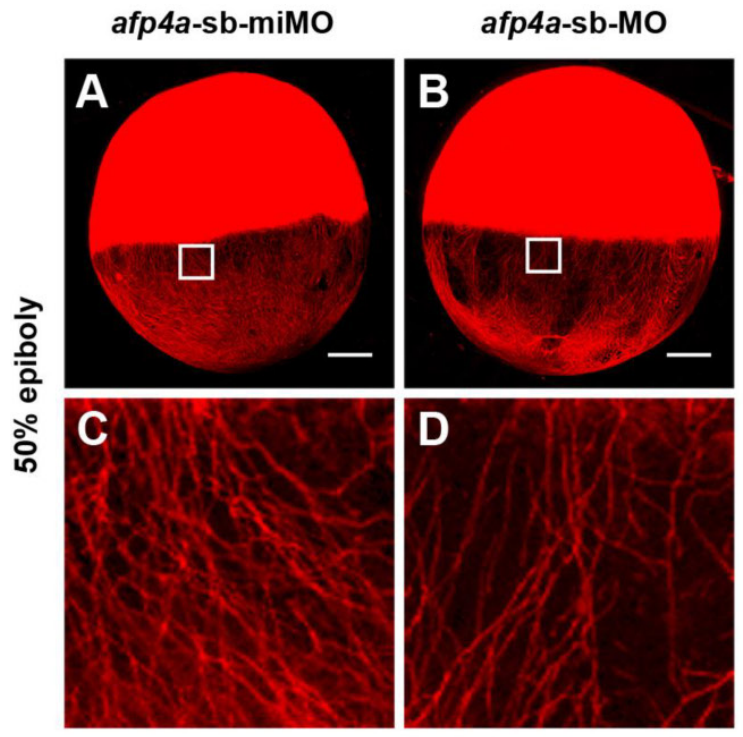

$\mathbf{E}$

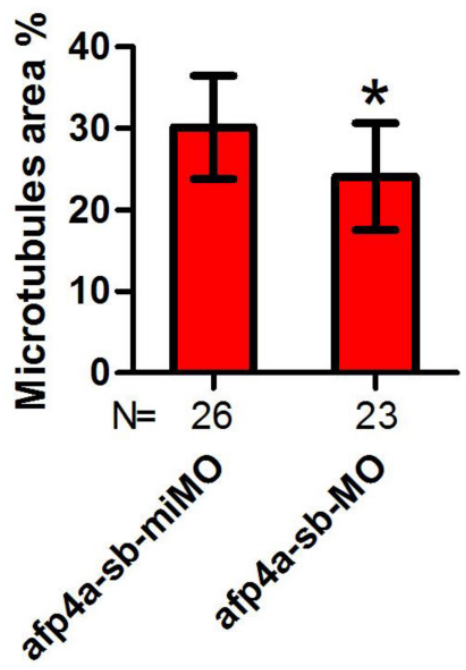

$\mathbf{F}$

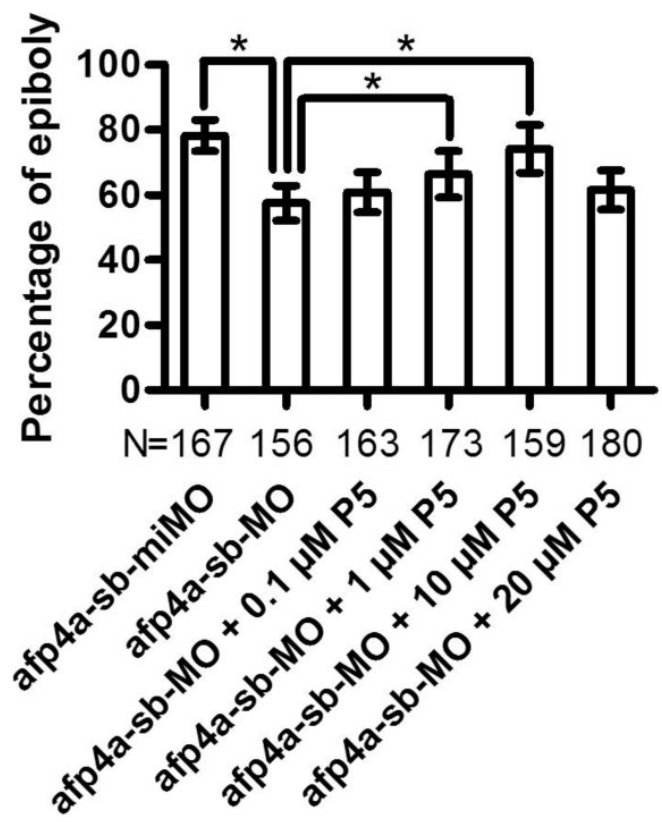

Figure 5. YCL microtubule arrays of afp 4a-sb-MO morphants are less stable than that of afp $4 a$-sb-miMO control embryos. (A-D) YCL microtubules are thinner in afp 4a-sb-MO embryos (B, D) compared to those in afp4a-sb-miMO embryos (A, C), at $50 \%$ epiboly after nocodazole treatment. ( $C$ or $D)$ is the enlarged image of the areas in the square in (A or B). Embryos are oriented with anterior up. Scale bars, 100 $\mu \mathrm{m}$. (E) Average percentage of the microtubule area in $\mathrm{YCL}$ alone EVL margin in afp $4 a$-sb-miMO embryos and afp $4 a$-sb-MO morphants at about $50 \%$ epiboly after nocodazole treatment. (F) Epibolic defect in AFP4a-deficient embryos can be rescued by $I$ and $10 \mu \mathrm{M}$ pregnenolone (P5). (E and F) Data are represented as means \pm SD. N, the number of embryos that were analyzed from three independent experiments. *P $<0.01$.

\section{AFP4a participates in epiboly progression by stabilizing YCL microtubules}

Above observed phenotypes in the afp $4 a$-sb-MO morphants, such as obviously delayed epiboly, mildly affected CE, and normal germ layer induction, involution and anterior axial formation, are highly similar to those of YCL microtubule disrupted embryos [6, 47]. This strong resemblance promoted us to visualize microtubule array in YCL by staining them with antibody against $\beta$-tubulin. Firstly, we compared the afp $4 a$-sb-miMO and afp $4 a$-sb-MO embryos fixed at $50 \%$ epiboly stage, and no any changes were observed (data not shown). Then, we treated the afp $4 a$-sb-miMO and afp $4 a$-sb-MO embryos at $50 \%$ epiboly stage with 1 $\mu \mathrm{g} / \mathrm{ml}$ nocodazole for $20 \mathrm{~min}$ at $28.5^{\circ} \mathrm{C}$, and examined the microtubules again in the fixed embryos. Obviously, the YCL microtubules in the afp $4 a$-sb-MO embryos were thinner than those in the afp $4 a$-sb-miMO embryos (Fig. 5A-D). To quantify the data, the percentages of microtubule area in $100 \times 100$ pixel images were obtained, and a statistical analysis was performed. The average percentage of microtubule area was $30.1 \%(\mathrm{SD}=6.3 \%, \mathrm{~N}=26)$ in the afp $4 a-\mathrm{sb}-\mathrm{miMO}$ embryos, whereas it was only $24.1 \%(\mathrm{SD}=6.6 \%, \mathrm{~N}=23)$ in the afp $4 a$-sb-MO morphants (Fig. 5E), indicating that the stability of microtubules is reduced in the afp $4 a$-sb-MO morphants.

To confirm that the epiboly delay resulted from the microtubule disruption, we used pregnenolone $(\mathrm{P} 5,0.1,1,10$ and $20 \mu \mathrm{M})$ to incubate the afp $4 a$-sb-MO morphants, since pregnenolone was demonstrated to promote epiboly by stabilizing microtubules [6]. Significantly, when afp $4 a$-sb-miMO embryos reached to $78.2 \%$ epiboly ( $\mathrm{SD}=4.8 \%, \mathrm{~N}=167)$, the blastoderms only covered $57.4 \%$ yolk cells $(\mathrm{SD}=5.4 \%, \mathrm{~N}=156)$ in the afp $4 a$-sb-MO morphants. In addition, the epibolic defect of afp $4 a$-sb-MO morphants could be partially rescued by the pregnenolone treatment, in which the significant rescuing increases were observed from 1 to $10 \mu \mathrm{M}$ pregnenolone treatment $(66.3 \% \pm 7.2 \%, \mathrm{~N}=173$; $74.1 \% \pm 7.3 \%, \mathrm{~N}=159)$. However, a rescuing decrease was observed in $20 \mu \mathrm{M}$ treatment $(61.4 \% \pm 6.0 \%$, $\mathrm{N}=180$ ) (Fig. 5F). A similar epibolic defect was also observed in embryos treated by high dose of microtubule stabilizing agent taxol [48]. In this respect, this 
decrease could be interpreted as a result of too stable microtubule arrays. These results indicate that YCL microtubule cytoskeleton is disturbed in the afp $4 a$ morphants, suggesting that AFP4a might participate in epiboly progression by stabilizing YCL microtubules.

Another actin cytoskeleton structure, including F-actin rings at the vegetal margin of deep cells and EVL, and punctuate actin band in the external-YSL lying vegetal to the EVL leading margin, which form after $50 \%$ epiboly, had been revealed to be an important drive of the second half epiboly [43]. To reveal whether the actin cytoskeleton was also affected in the afp $4 a$ morphants, we performed F-actin staining with rhodamine-phalloidin. In afp $4 a$-sb-miMO embryos, the punctuate actin band initiated to form at $6 \mathrm{hpf}$ and afp4a-sb-miMO
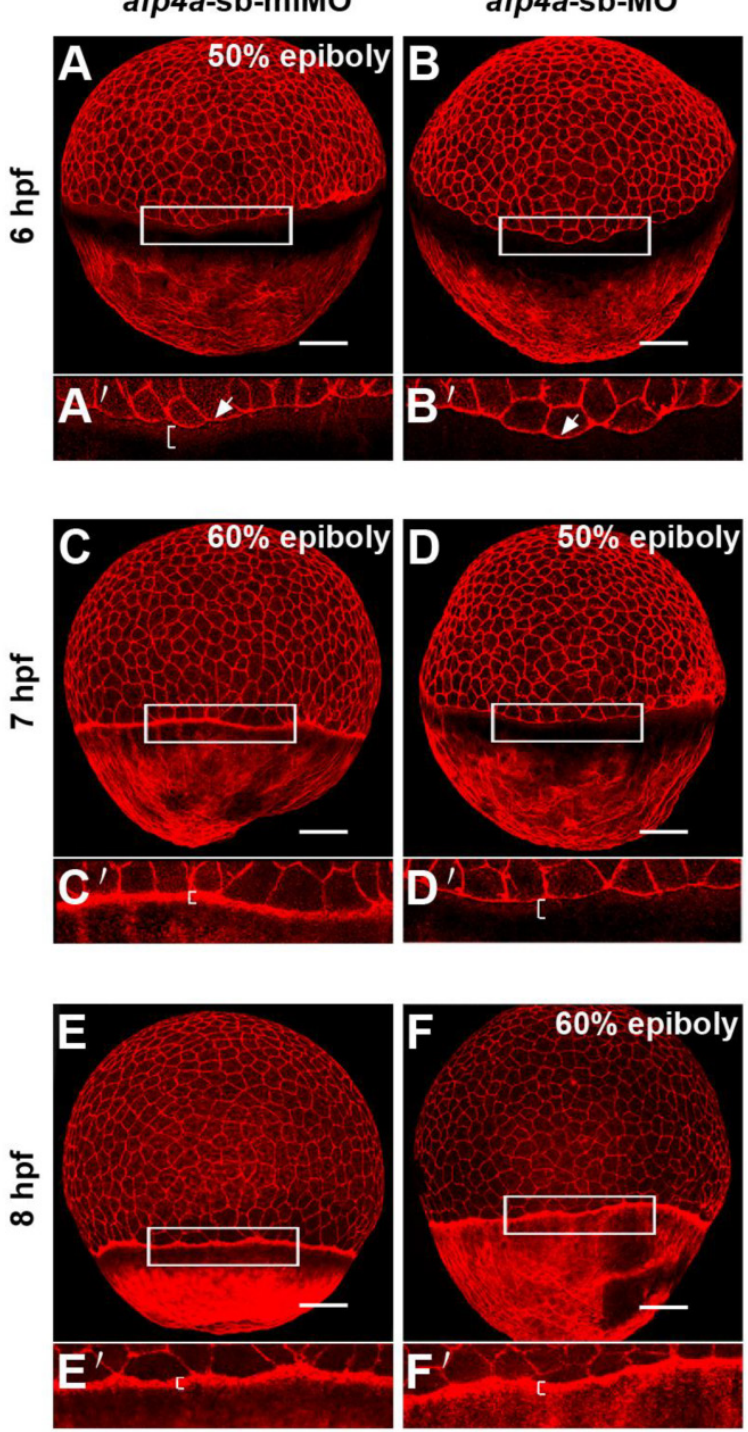

became very clear at $7 \mathrm{hpf}$ (Fig. 6A, C and E), whereas in the afp $4 a$-sb-MO morphants, it began to appear at 7 hpf and displayed obviously at $8 \mathrm{hpf}$ (Fig. 6B, D and F). This difference might be resulted from epiboly progression delay. Quantitative analyses indicated that there were obviously different phalloidin signals between the afp $4 a$-sb-miMO embryos and the afp $4 a$ morphants at their corresponding $6 \mathrm{hpf}$ and $7 \mathrm{hpf}$ (Fig. 6G, H), but basic similar phalloidin intensity signals were observed from their same development stages, when $50 \%$ or $60 \%$ epiboly embryos were comparatively analyzed (Fig. 6I). The results suggest that the actin cytoskeleton should be not affected in the afp $4 a$ morphants, and AFP4a might specifically interact with YCL microtubules to participate in epiboly progression.

\section{$\mathbf{G}$}

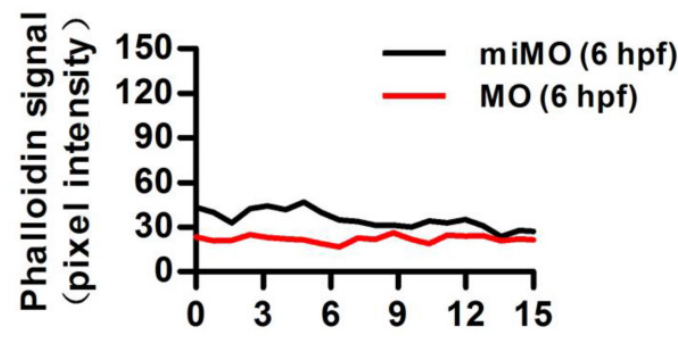

Distance from leading edge $(\mu \mathrm{m})$
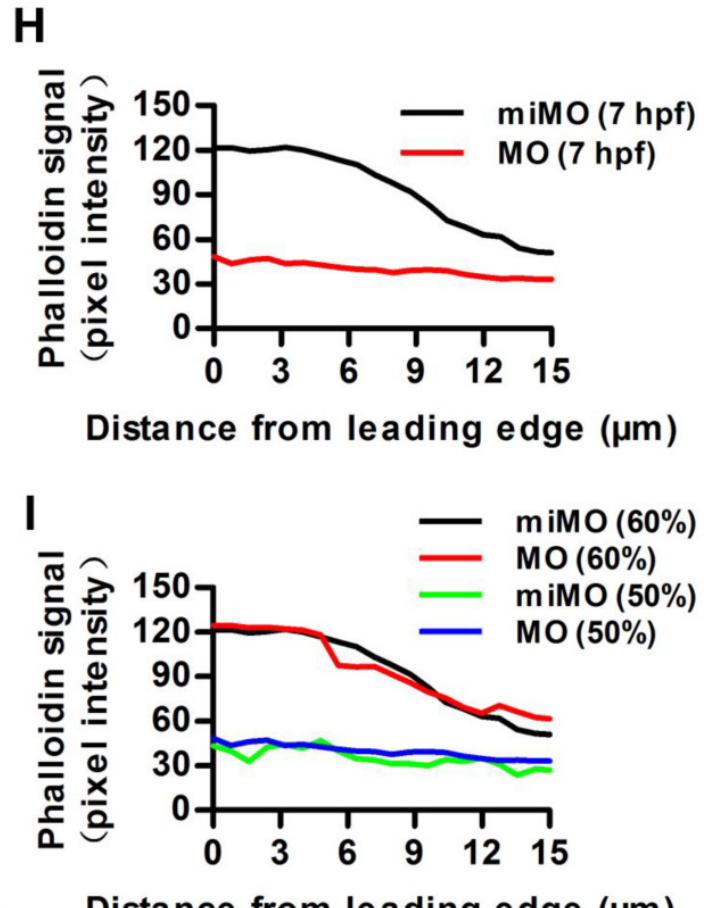

Distance from leading edge $(\mu \mathrm{m})$

Figure 6. Formation of the punctate actin band in YSL seems to be timed by an epiboly-dependent clock in afp4a-sb-MO morphants. (A-F) afp4a-sb-miMO embryos (A, C, E) and the same-age afp4a-sb-MO embryos (B, D, F) at $6 \mathrm{hfp}(\mathrm{A}, \mathrm{B}), 7 \mathrm{hpf}(\mathrm{C}, \mathrm{D})$ and $8 \mathrm{hpf}(\mathrm{E}, \mathrm{F})$ stained with rhodamine-phalloidin (F-actin). Among them, afp4a-sb-miMO morphants at $6 \mathrm{hpf}(\mathrm{A})$ and afp $4 a$-sb-MO morphants at $7 \mathrm{hpf}(\mathrm{D})$ reach about $50 \%$ epiboly, and afp $4 a$-sb-miMO morphants at 7 hpf (C) and afp $4 a-s b-M O$ morphants at 8 hpf (F) reach about $60 \%$ epiboly. $\left(A^{\prime}-F^{\prime}\right)$ are the enlarged images of the areas in the rectangle in corresponding (A-F). Embryos are oriented with their animal pole up; brackets in $A^{\prime}-F^{\prime}$ indicate the punctuate actin bands; white arrows in $\mathrm{A}^{\prime}$ and $\mathrm{B}^{\prime}$ indicate leading edge of the EVL; scale bars, I00 $\mu \mathrm{m}$. (G-I) Average intensity of phalloidin signal in the YSL along the EVL margin in afp4a-sb-miMO (miMO) embryos and afp4a-sb-MO (MO) morphants at 6 hpf (G), 7 hpf (H) and 50\% and 60\% epiboly (I). The intensity of the phalloidin signal was plotted along a line perpendicular to the EVL margin. Average plots of ten embryos for each group are shown. 


\section{AFP4b is required for CE movement without affecting dorsoventral axis patterning}

To investigate the role of AFP4b in embryogenesis, we synthesized a 5-base mismatched control $\mathrm{MO}$ to $a f p 4 b$-tb-MO (afp $4 b$-tb-miMO), and observed a normal embryo phenotype similar to the Ctrl-MO injection at same concentration of $2.5 \mathrm{ng} /$ embryo (data not shown). So, the afp $4 b$-tb-miMO was served as a control of the $a f p 4 b$-tb-MO in subsequent research about the function of AFP4b. In comparison with control afp $4 b$-tb-miMO embryos, the somite width was significantly enhanced in the $a f p 4 b$-tb-MO morphants at 8 -somite stage, as revealed by myoD probe. This abnormity could be rescued only by co-injection of $150 \mathrm{pg} a f p 4 b$-mRNA, but not by the same amount of afp 4a-mRNA (Fig. 7A). A quantitative and statistical analysis showed a significant increase of average somite width $(251.4 \pm 45.2 \mu \mathrm{m}, \mathrm{N}=176, \mathrm{P}<0.01)$ in the $a f p 4 b$-tb-MO morphants relative to that in the
afp4b-tb-miMO embryos $(192.0 \pm 10.3 \mu \mathrm{m}, \mathrm{N}=160)$. Moreover, this increase could be effectively recovered by the afp $4 b$-mRNA co-injection (195.6 $\pm 40.1 \mu \mathrm{m}$, $\mathrm{N}=149$ ), but could not be rescued by the same amount of afp $4 a$-mRNA (245.6 $\pm 37.6 \mu \mathrm{m}, \mathrm{N}=153)$ (Fig. 7B). These data indicate that the somite defect is resulted from specific reduction of AFP4b expression.

The above somite defect implied that AFP4b might be involved in CE movements, because it was very similar to the previous observation in has 2 and rhoA morphants $[49,50]$. For this reason, we firstly used dorsal $(g s c)$ and ventral (eve1) makers to detect dorsoventral patterning change in the afp $4 b$-tb-MO morphants. In comparison with afp $4 b$-tb-miMO embryos, no any obvious change was observed in the $a f p 4 b$-tb-MO morphants at 75\% epiboly (Fig. 7C, D), indicating that AFP4b knockdown did not disrupt dorsoventral axis patterning and differentiation.
A
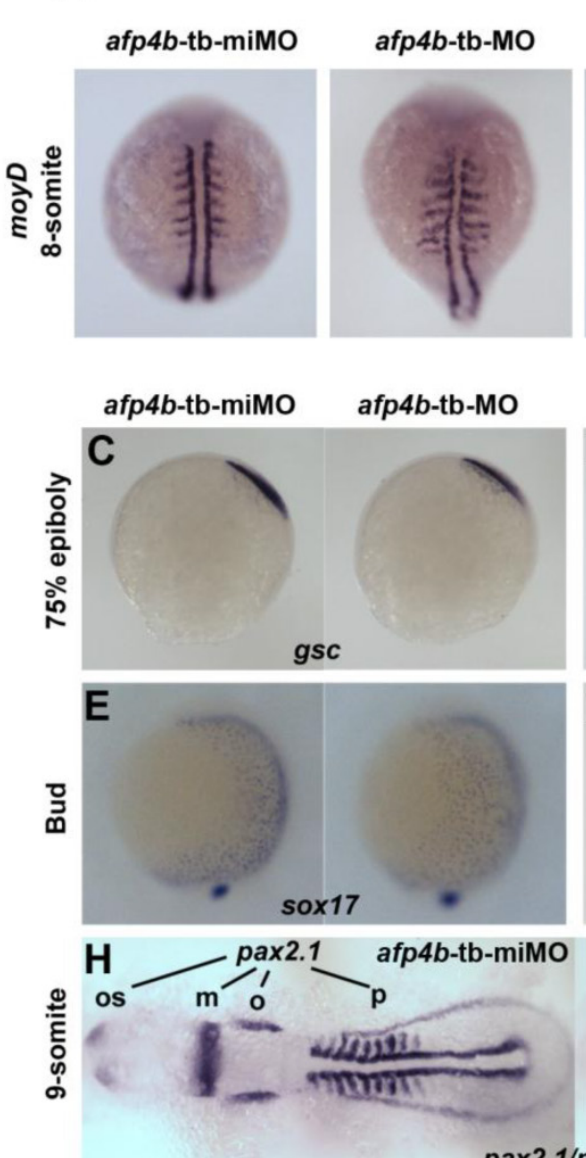

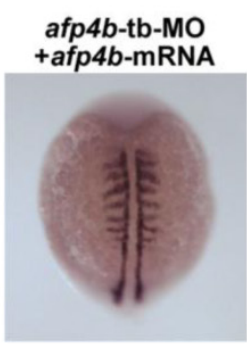

afp $4 b$-tb-miMO
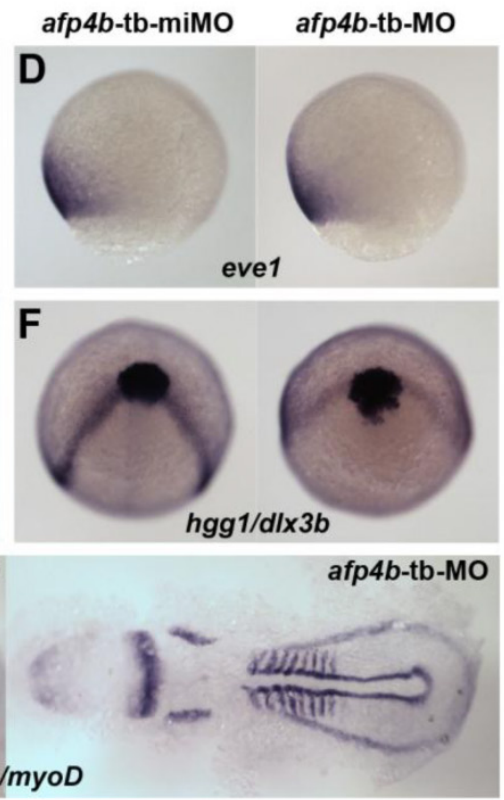
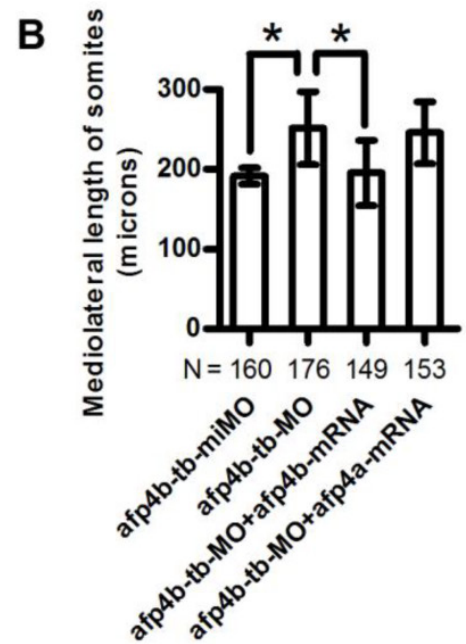

afp4b-tb-miMo afp4b-tb-MO
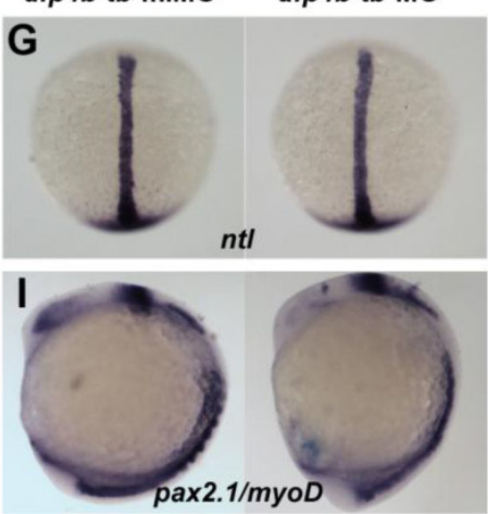

Figure 7. Reduced AFP4b expression affects CE movement. (A) myoD expression in 8-somite groups injected with afp4b-tb-miMO, afp4b-tb-MO, afp4b-tb-MO plus afp $4 b$-mRNA, or afp $4 b$-tb-MO plus afp 4a-mRNA. Dorsal views of embryos, anterior to the top. (B) Average mediolateral length of somites in each group. Error bars show SD. $\mathrm{N}$, the number of embryos that were analyzed from three independent experiments. $* \mathrm{P}<0.0 \mathrm{l}$. (C-I) Dorsoventral pattern is normal, but CE is affected in afp $4 b$-tb-MOs morphants showing by whole-mount in situ hybridization. In each section, expression of marker genes in afp $4 b$-tb-miMO morphants (left) and afp $4 b$-tb-MO morphants (right) are shown. (C) gsc, $75 \%$ epiboly stage. (D) evel, 75\% epiboly stage. (E) sox I 7, bud stage. (C-E) Lateral views, dorsal to the right. (F) hggl and dlx3b, bud stage, top view, ventral up. (G) $n t$, bud stage, dorsal view, animal pole to the top. ( $\mathrm{H}$ and I) pax2.I and myoD, 9-somite stage. $(\mathrm{H})$ Flat-mounted embryos, head to the left. os, optic stalk; m, the midbrain hindbrain boundary; o, otic vesicles; p, pronephros. (I) Lateral view, dorsal to the right. 
Moreover, we detected CE movements by marker genes specific to endoderm (sox17), axial and paraxial mesoderm $(h g g 1, n t l, m y o D)$, and neuroectoderm ( $d l \times 3 b$ and pax2.1). The sox17-labeled endoderm cells moved slower dorsally during gastrulation in $a f p 4 b$-tb-MO morphants, as indicated by the earlier dorsoventral expression pattern of sox17 at the end of gastrulation (Fig. 7E). Thus, the CE defect in $a f p 4 b$-tb-MO morphants might be associated with dorsal migration of ventrolateral endoderm cells. In addition, the neural plate was much wider in $a f p 4 b$-tb-MO morphants, as reflected by the laterally expansion expression domain of $d l x 3 b$ at bud stage (Fig. 7F) and pax2.1 (the midbrain hindbrain boundary, the otic vesicles) at 9-somite stage (Fig. $7 \mathrm{H})$. Thus, the CE movement of neuroectoderm was delayed in $a f p 4 b$-tb-MO morphants. Moreover, afp $4 b$-tb-MO morphants showed indistinguishable notochord from control embryos, as revealed by the expression of $n t l$ at bud stage (Fig. 7G). However, afp $4 b$ morphants displayed a broader somatic mesoderm and short head to tail distance during somitogenesis, as represented by the expression patterns of myoD and pax2.1 (the optic stalk to the posterior end of the pronephros) in Flat-mounted embryos at 9-somite stage (Fig. 7H). At the same time, afp $4 b$-tb-MO morphants still showed a longer anterior-posterior axis (Fig. 7I). Similar observations were also reported in research of has2 and npc1 [7, 49]. Therefore, the CE movement of axial mesoderm was least affected, but that of paraxial

mesoderm was significantly changed in afp $4 b$-tb-MO morphants. The above data indicate that CE movement of ventrolateral cells is severely impaired, whereas CE movement of axial cells and dorsoventral axis patterning are normal after the AFP4b knockdown, implying that AFP4b is required for CE movement without affecting dorsoventral axis patterning.

\section{AFP4b is primarily related to convergence}

To directly clarify how CE movements are affected by AFP4b depletion during gastrulation, we respectively injected Kaede mRNA with $a f p 4 b$-tb-miMO or afp $4 b$-tb-MO into one-cell stage embryos. Then, Kaede protein was activated from green to a bright and stable red fluorescence by UV illumination at a specific time and in a specific cell group, and cell movements were traced as previously described [41]. Firstly, the lateral marginal cells, $90^{\circ}$ from the dorsal shield, were activated at $6 \mathrm{hpf}$ by a focused UV pulse (Fig. 8A and D), and the movement trajectories of the labeled red fluorescence cells were traced. In comparison with normal movement trajectories in $a f p 4 b$-tb-miMO embryos (Fig. 8B and C), the labeled red fluorescence cells in the afp $4 b$-tb-MO embryos underwent normal animal and vegetal migrations, but the movement towards the dorsal side was severely affected, and the labeled cells did not reach to the midline (Fig. 8E and F).

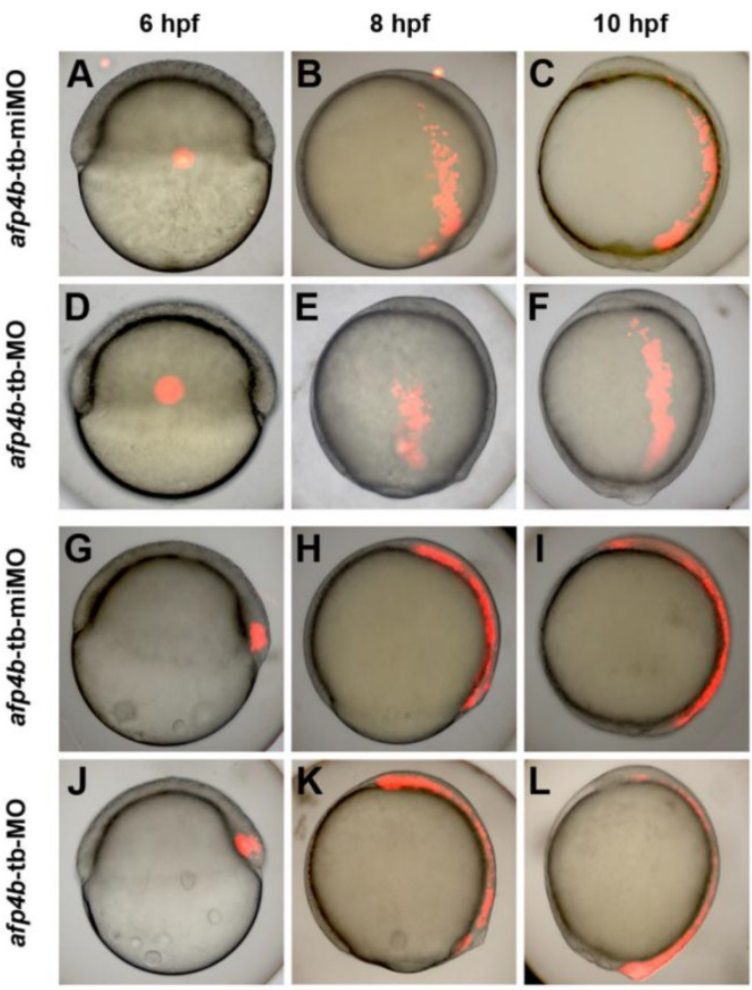

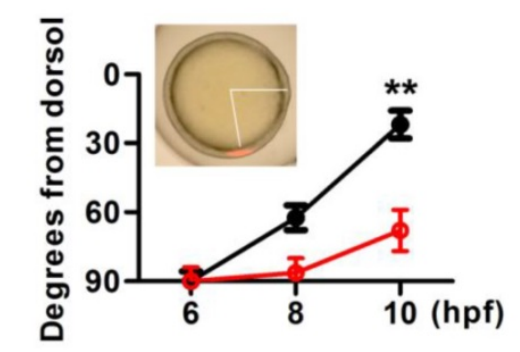

M

$\mathbf{N}$

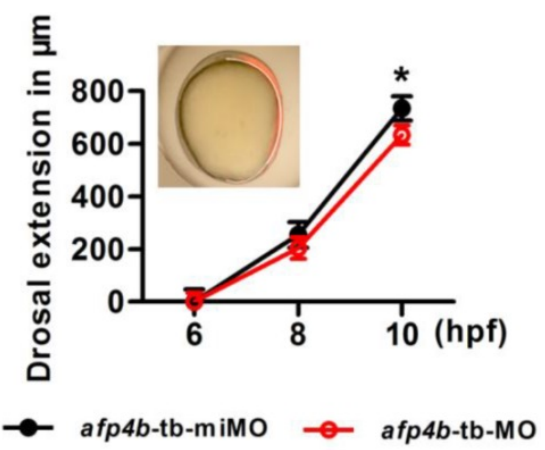

Figure 8. Cell tracing experiments. (A-F) Distribution of labeled lateral cells in afp $4 b$-tb-miMO embryos (A-C) and afp $4 b$-tb-MO embryos (D-F) at $6 \mathrm{hpf}(\mathrm{A}$, $D), 8 \mathrm{hpf}(\mathrm{B}, \mathrm{E})$ and $10 \mathrm{hpf}(\mathrm{C}$, F). (G-L) Distribution of labeled dorsal cells in afp $4 b$-tb-miMO embryos (G-I) and afp $4 b$-tb-MO embryo (J-L) at 6 hpf (G, J), 8 hpf $(\mathrm{H}, \mathrm{K})$ and $10 \mathrm{hpf}(\mathrm{I}, \mathrm{L})$. (A-L) Lateral views, animal pole is up and dorsal is to the right. (M) Graph displaying the average degree from the dorsal axis to labeled cells (indicated by angle) at $6 \mathrm{hpf}, 8$ hpf and $10 \mathrm{hpf}$, **P $<0.00 \mathrm{I}$. (N) Graph displaying the average extension in labeled dorsal cells (indicated by arc) at $6 \mathrm{hpf}, 8 \mathrm{hpf}$ and $10 \mathrm{hpf}$, *P < 0.05 . Ten embryos per treatment were evaluated, and error bars show SD. 
A quantitative and statistical analysis showed a significant difference of distance $(\mathrm{P}<0.001)$ from dorsal axis between $a f p 4 b$-tb-miMO $\left(22 \pm 6^{\circ}\right)$ embryos and $a f p 4 b$-tb-MO morphants $\left(68 \pm 9^{\circ}\right)$ at $10 \mathrm{hpf}$ (Fig. $8 \mathrm{M})$, implicating that AFP4b might be related to convergent movement of lateral cells towards the dorsal side.

To measure the relationship between AFP4b and dorsal extension movement, a group of cells within the dorsal embryonic shield was marked at 6 hpf (Fig. $8 \mathrm{G}, \mathrm{J})$, and the movement of labeled cells was recorded at $8 \mathrm{hpf}$ and $10 \mathrm{hpf}$. In both the $a f p 4 b$-tb-miMO embryos and the afp $4 b$-tb-MO morphants at $10 \mathrm{hpf}$, the marked cells were similarly distributed in the dorsal axial cells, and only a slight dorsal extension delay was observed in the afp $4 b$-tb-MO morphants (Fig. 8G-L). A quantitative and statistical analysis also showed a slight reduction $(\mathrm{P}<0.05)$ in the dorsal extension of the afp $4 b$-tb-MO morphants $(634 \pm 36 \mu \mathrm{m})$ relative to the afp $4 b$-tb-miMO embryos (735 $\pm 46 \mu \mathrm{m})$ (Fig. 8N). Together, these data indicate that AFP4b is primarily related to convergence movement.

\section{Discussion}

In this study, we have identified two head-to-tail tandem duplicated afp 4 genes (afp $4 a$ and $a f p 4 b$ ) from gibel carp and zebrafish, and found that they possess similar genomic structures and protein sequences. Comparison of cDNA sequences between afp $4 a$ and $a f p 4 b$ shows high similarity in their ORF sequences, whereas significant differences exist in their UTR sequences and promoter sequences. RT-PCR analysis and WISH have revealed differential expression patterns and dynamic changes between afp $4 a$ and $a f p 4 b$ during embryogenesis, early larval development and adults. Thereby, we have found for the first time that both $a f p 4 a$ and $a f p 4 b$ are specifically expressed in YSL, but the later expressed $a f p 4 b$ exists only in embryogenesis, whereas afp $4 a$ expresses continuously in YSL and digestive system from early embryos to adults. Subsequently, we have studied the roles of AFP4a and AFP4b by using afp $4 a$-specific and $a f p 4 b$-specifc morpholino knockdown approaches, and found that AFP4a and AFP4b contribute to early morphogenetic movements during zebrafish embryogenesis. Moreover, we have observed that the YCL microtubule cytoskeleton is disturbed in the afp $4 a$ morphants, while the actin cytoskeleton is not affected in the afp $4 a$ morphants, suggesting that AFP4a might participate in epiboly progression by stabilizing YCL microtubules. In addition, we have revealed that AFP4b is required for $\mathrm{CE}$ movement without affecting dorsoventral axis patterning, and demonstrated that AFP4b is primarily related to convergence movement. Therefore, this current study has confirmed that both
AFP4a and AFP4b are key regulators during zebrafish embryogenesis, and contribute to epiboly progression and convergence movement, respectively.

AFP4 was firstly isolated from the serum of longhorn sculpin in 1997 [23], and genomic structure of afp 4 had not been described until 2011 by Lee et al [25]. Previously, two afp4 homologs have been reported from databases of three-spined stickleback (Gasterosteus aculeatus) and Atlantic salmon (Salmo salar) [25], but their distributions in the chromosomes have not been clarified. Here, for the first time, we have shown the genomic organization and the head-to-tail tandem distribution of the two duplicated afp $4 \mathrm{~s}$ in gibel carp and zebrafish. Gene duplication has been believed to be a major evolutionary driving force for organism complexity, and expression divergence has been proposed as the first step of functional divergence between duplicate genes [51]. The afp $4 a$ and $a f p 4 b$ are highly similar in their ORFs, but significant differences are found in their UTR sequences and promoter sequences, and differential expression patterns between afp $4 a$ and $a f p 4 b$ are also observed during their embryogenesis and early larval development. These differences should be consistent with their functional divergence. Previous studies have demonstrated that the cis-regulatory elements in the promoters can result in expression divergence [52] and the divergent sequences in $3^{\prime}$ UTR also affect the expression pattern of duplicate genes $[53,54]$.

Along with AFP4s have been identified in temperate, subtropical and tropical fishes that have no need to prevent freezing [20, 24, 25], their biological roles have been speculated to bind to lipid or ligand other than ice because of the helix bundle structure similarity to certain apolipoproteins (Apos) [24, 26, 28], but the exact physiological functions have been unknown up to the present. Our current study has revealed the biological roles of AFP4a and AFP4b in embryonic development, and has confirmed that AFP4a and AFP4b contribute to epiboly progression and convergence movement during early zebrafish embryogenesis. Recently, some Apos, such as Apo-14 [55, 56], ApoA-II [57], ApoB [58], ApoC1 [45], have been also reported to participate in embryonic morphogenesis and organogenesis in gibel carp and zebrafish. Significantly, homological searches also show about $20 \%$ identities between AFP4s and some Apos that include mammalian ApoA-II and fish Apo-14, and the conserved amphipathic alpha-helices within AFP4s are also a common property in Apos. Therefore, we propose that afp4s might share a common ancestral gene with some apos genes, and still maintain the original function in temperate, subtropical and tropical fishes. In addition, afp $4 a$ and $a f p 4 b$ show similar expression patterns with Apo-14 [55] 
and ApoC1 [45] during embryogenesis, and all of them are expressed in YSL. As it has been previously reported that some genes expressed in YSL function during early embryonic morphogenesis [45, 55, 57-60], AFP4a and AFP4b also play the related roles.

Another significant finding in this study is about the association between AFP4a and YCL microtubule cytoskeleton, because our results have revealed that the afp $4 a-\mathrm{MO}$ morphants display an obvious defect in epiboly progression, and YCL microtubule arrays are less stable in afp $4 a-\mathrm{MO}$ morphants. Previous studies have indicated that microtubule arrays in YCL are important drive of epiboly, and their homeostasis is crucial for normal epibolic movement [47, 48]. Two groups of related factors have been identified. The first group, which can stabilize YCL microtubules, includes Cyp11a1 [6] and calcium channel $\beta 4$ subunits (CACNB4) [60]. The second group, which are involved in microtubules organization, includes pou domain, class 5, transcription factor 1 (Pou5f1) [61] and eomesodermin A (Eomesa) [62]. Of these epiboly mutants and morphants, afp $4 a-\mathrm{MO}$ morphants most closely resemble cyp11a1-MO morphants. Firstly, afp $4 a$ and cyp11a1 have the common expression domain in YSL. Secondly, they both display serious epibolic defect and slight $\mathrm{CE}$ defect, but do not change germ layer patterning and involution. Thirdly, stability of YCL microtubules is lowered in both embryos. In addition, pregnenolone, the catalytic product of Cyp11a1, can partially rescue the epibolic defect of AFP4a-knockdown embryos. Our study indicates that AFP4a either directly or indirectly stabilizes the microtubules in YCL, which extends our knowledge of YCL microtubule regulation.

\section{Supplementary Material}

Figures S1 - S4, Tables S1 - S2.

http://www.ijbs.com/v10p0715s1.pdf

\section{Abbreviations}

AFP: Antifreeze protein; AFP4: type-IV antifreeze proteins; afp4: type-IV antifreeze protein gene; Cag: Carassius auratus gibelio; Dr: Danio rerio; Cagafp4: afp4 in gibel carp; Drafp4s: afp4s in zebrafish; YSL: yolk syncytial layer; YCL: yolk cytoplasmic layer; CE: convergence and extension; RACE: rapid amplification of cDNA ends; WT: wild type; qPCR: Real-time PCR; ML: maximum likelihood; WISH: whole-mount in situ hybridization; SD: standard deviation; ANOVA: analysis of variance; UTR: untranslated region; ORF: open reading frame; UV: ultraviolet; hpf: hours postfertilization; EVL: enveloping layer; Apo: apolipoprotein.

\section{Acknowledgements}

This work was funded by the National Key Basic Research Program of China (2010CB126301), the Strategic Priority Research Program of the Chinese Academy of Sciences (XDA08030201), the earmarked fund for Modern Agro-industry Technology Research System (NYCYTX-49), the Innovation Project of Chinese Academy of Sciences (KSCX3-EW-N-04), and the Autonomous Project of the State Key Laboratory of Freshwater Ecology and Biotechnology (2011FBZ17).

\section{Competing Interests}

The authors have declared that no competing interest exists.

\section{References}

1. Keller R. Shaping the vertebrate body plan by polarized embryonic cell movements. Science. 2002; 298: 1950-4.

2. Kimmel CB, Ballard WW, Kimmel SR, Ullmann B, Schilling TF. Stages of embryonic development of the zebrafish. Dev Dyn. 1995; 203: 253-310.

3. Solnica-Krezel L, Sepich DS. Gastrulation: making and shaping germ layers. Annu Rev Cell Dev Biol. 2012; 28: 687-717.

4. Solnica-Krezel L. Gastrulation in zebrafish - all just about adhesion? Curr Opin Genet Dev. 2006; 16: 433-41.

5. Rohde LA, Heisenberg CP. Zebrafish gastrulation: cell movements, signals, and mechanisms. Int Rev Cytol. 2007; 261: 159-92.

6. Hsu HJ, Liang MR, Chen CT, Chung BC. Pregnenolone stabilizes microtubules and promotes zebrafish embryonic cell movement. Nature. 2006; 439: 480-3.

7. Schwend T, Loucks EJ, Snyder D, Ahlgren SC. Requirement of Npc1 and availability of cholesterol for early embryonic cell movements in zebrafish. J Lipid Res. 2011; 52: 1328-44.

8. Yin C, Ciruna B, Solnica-Krezel L. Convergence and extension movements during vertebrate gastrulation. Curr Top Dev Biol. 2009; 89: 163-92.

9. Dong CH, Yang ST, Yang ZA, Zhang L, Gui JF. A C-type lectin associated and translocated with cortical granules during oocyte maturation and egg fertilization in fish. Dev Biol. 2004; 265: 341-54.

10. Peng JX, Xie JL, Zhou L, Hong YH, Gui JF. Evolutionary conservation of Dazl genomic organization and its continuous and dynamic distribution throughout germline development in gynogenetic gibel carp. J Exp Zool Part B (Mol Dev Evol). 2009; 312B: 855-71.

11. Wu N, Yue HM, Chen B, Gui JF. Histone H2A has a novel variant in fish oocytes. Biol Reprod. 2009; 81: 275-83.

12. Yue HM, Li Z, Wu N, Liu Z, Wang Y, Gui JF. Oocyte-specific H2A variant H2af1o is required for cell synchrony before mid-blastula transition in early zebrafish embryos. Biol Reprod. 2013; 89: 82.

13. Gui JF, Zhu ZY. Molecular basis and genetic improvement of economically important traits in aquaculture animals. Chin Sci Bull. 2012; 57: 1751-60.

14. Zhou L, Wang Y, Gui JF. Genetic evidence for gonochoristic reproduction in gynogenetic silver crucian carp (Carassius auratus gibelio Bloch) as revealed by RAPD assays. J Mol Evol. 2000; 51: 498-506.

15. Yang L, Gui JF. Positive selection on multiple antique allelic lineages of transferrin in the polyploid Carassius auratus. Mol Biol Evol. 2004; 21: 1264-77.

16. Gui JF, Zhou L. Genetic basis and breeding application of clonal diversity and dual reproduction modes in polyploid Carassius auratus gibelio. Sci China Life Sci. 2010; 53: 409-15.

17. Wang ZW, Zhu HP, Wang D, Jiang FF, Guo W, Zhou L, et al. A novel nucleo-cytoplasmic hybrid clone formed via androgenesis in polyploid gibel carp. BMC Res notes. 2011; 4: 82.

18. Jiang FF, Wang ZW, Zhou L, Jiang L, Zhang XJ, Apalikova OV, et al. High male incidence and evolutionary implications of triploid form in northeast Asia Carassius auratus complex. Mol Phylogenet Evol. 2013; 66: 350-9.

19. Zhai YH, Zhou L, Wang Y, Wang ZW, Li Z, Zhang XJ, et al. Proliferation and resistance difference of a liver-parasitized myxosporean in two different gynogenetic clones of gibel carp. Parasitol Res. 2014; 113: 1331-41.

20. Liu JX, Zhai YH, Gui JF. Molecular characterization and expression pattern of AFPIV during embryogenesis in gibel carp (Carassiu auratus gibelio). Mol Biol Rep. 2009; 36: 2011-8.

21. Doxey AC, Yaish MW, Griffith M, McConkey BJ. Ordered surface carbons distinguish antifreeze proteins and their ice-binding regions. Nat Biotechnol. 2006; 24: 852-5.

22. Ewart $\mathrm{K}$, Lin Q, Hew C. Structure, function and evolution of antifreeze proteins. Cell Mol Life Sci. 1999; 55: 271-83.

23. Deng G, Andrews DW, Laursen RA. Amino acid sequence of a new type of antifreeze protein, from the longhorn sculpin Myoxocephalus octodecimspinosis. FEBS Lett. 1997; 402: 17-20. 
24. Gauthier SY, Scotter AJ, Lin FH, Baardsnes J, Fletcher GL, Davies PL A re-evaluation of the role of type IV antifreeze protein. Cryobiology. 2008; 57: 292-6.

25. Lee JK, Kim YJ, Park KS, Shin SC, Kim HJ, Song YH, et al. Molecular and comparative analyses of type IV antifreeze proteins (AFPIVs) from two Antarctic fishes, Pleuragramma antarcticum and Notothenia coriiceps. Comp Biochem Physiol B Biochem Mol Biol. 2011; 159: 197-205.

26. Deng G, Laursen RA. Isolation and characterization of an antifreeze protein from the longhorn sculpin, Myoxocephalus octodecimspinosis. Biochim Biophys Acta. 1998; 1388: 305-14.

27. Goetz FW, McCauley L, Goetz GW, Norberg B. Using global genome approaches to address problems in cod mariculture. Ices J Mar Sci. 2006; 63: 393-9.

28. Breton TS, Anderson JL, Goetz FW, Berlinsky DL. Identification of ovarian gene expression patterns during vitellogenesis in Atlantic cod (Gadus morhua). Gen Comp Endocr. 2012; 179: 296-304

29. Celik Y, Drori R, Pertaya-Braun N, Altan A, Barton T, Bar-Dolev M, et al. Microfluidic experiments reveal that antifreeze proteins bound to ice crystals suffice to prevent their growth. Proc Natl Acad Sci USA. 2013; 110: 1309-14.

30. Fletcher GL, Hew CL, Davies PL. Antifreeze proteins of teleost fishes. Annu Rev Physiol. 2001; 63: 359-90.

31. Cheng C-HC, Detrich HW. Molecular ecophysiology of Antarctic notothenioid fishes. Philos T Roy Soc B. 2007; 362: 2215-32.

32. Geng FS, Zhou L, Gui JF. Construction and characterization of a BAC library for Carassius auratus gibelio, a gynogenetic polyploid fish. Anim Genet. 2005; 36: 535 .

33. Xu H, Lim M, Dwarakanath M, Hong Y. Vasa identifies germ cells and critical stages of oogenesis in the Asian seabass. Int J Biol Sci. 2014; 10: 225-35.

34. Tamura K, Stecher G, Peterson D, Filipski A, Kumar S. MEGA6: molecular evolutionary genetics analysis version 6.0. Mol Biol Evol. 2013; 30: 2725-9.

35. Abascal F, Zardoya R, Posada D. ProtTest: selection of best-fit models of protein evolution. Bioinformatics. 2005; 21: 2104-5.

36. Westerfield M. The zebrafish book: a guide for the laboratory use of zebrafish (Danio rerio). Eugene: University of Oregon Press; 1995.

37. Huang W, Zhou L, Li Z, Gui JF. Expression pattern, cellular localization and promoter activity analysis of ovarian aromatase (Cyp19a1a) in protogynous hermaphrodite red-spotted grouper. Mol Cell Endocrinol. 2009; 307: 224-36.

38. Mei J, Zhang QY, Li Z, Lin S, Gui JF. C1q-like inhibits p53-mediated apoptosis and controls normal hematopoiesis during zebrafish embryogenesis. Dev Biol. 2008; 319: 273-84.

39. Mei J, Yue HM, Li Z, Chen B, Zhong JX, Dan C, et al. C1q-like factor, a target of miR-430, regulates primordial germ cell development in early embryos of Carassius auratus. Int J Biol Sci. 2014; 10: 15-24.

40. Li Z, Korzh V, Gong Z. Localized rbp4 expression in the yolk syncytial layer plays a role in yolk cell extension and early liver development. BMC Dev Biol. 2007; 7: 117

41. Lou QY, He JY, Hu L, Yin Z. Role of $l b x 2$ in the noncanonical Wnt signaling pathway for convergence and extension movements and hypaxial myogenesis in zebrafish. BBA-Mol Cell Res. 2012; 1823: 1024-32.

42. Zhong JX, Zhou L, Li Z, Wang Y, Gui JF. Zebrafish Noxa promotes mitosis in early embryonic development and regulates apoptosis in subsequent embryogenesis. Cell Death Differ. 2014; 21: 1013-24.

43. Cheng JC, Miller AL, Webb SE. Organization and function of microfilaments during late epiboly in zebrafish embryos. Dev Dyn. 2004; 231: 313-23.

44. Köppen M, Fernandez BG, Carvalho L, Jacinto A, Heisenberg CP. Coordinated cell-shape changes control epithelial movement in zebrafish and Drosophila. Development. 2006; 133: 2671-81.

45. Wang Y, Zhou L, Li Z, Li WH, Gui JF. Apolipoprotein C1 regulates epiboly during gastrulation in zebrafish. Sci China Life Sci. 2013; 56: 975-84.

46. Carvalho L, Heisenberg CP. The yolk syncytial layer in early zebrafish development. Trends Cell Biol. 2010; 20: 586-92.

47. Strahle U, Jesuthasan S. Ultraviolet irradiation impairs epiboly in zebrafish embryos: evidence for a microtubule-dependent mechanism of epiboly. Development. 1993; 119: 909-19.

48. Solnica-Krezel L, Driever W. Microtubule arrays of the zebrafish yolk cell: organization and function during epiboly. Development. 1994; 120: 2443-55.

49. Bakkers J, Kramer C, Pothof J, Quaedvlieg NEM, Spaink HP, Hammerschmidt M. Has2 is required upstream of Rac1 to govern dorsal migration of lateral cells during zebrafish gastrulation. Development. 2004; 131: 525-37.

50. Zhu S, Liu L, Korzh V, Gong Z, Low BC. RhoA acts downstream of Wnt5 and Wnt11 to regulate convergence and extension movements by involving effectors Rho Kinase and Diaphanous: Use of zebrafish as an in vivo model for GTPase signaling. Cell Signal. 2006; 18: 359-72.

51. Ohno S. Evolution by gene duplication. New york: Springer-Verlag; 1970.

52. Li J, Yuan Z, Zhang Z. Revisiting the contribution of cis-elements to expression divergence between duplicated genes: the role of chromatin structure. Mol Biol Evol. 2010; 27: 1461-6.

53. Tong Y, Zheng K, Zhao SF, Xiao GX, Luo C. Sequence divergence in the 3'-untranslated region has an effect on the subfunctionalization of duplicate genes. J Exp Zool Part B (Mol Dev Evol). 2012; 318B: 531-44.

54. Decembrini S, Andreazzoli M, Vignali R, Barsacchi G, Cremisi F. Timing the generation of distinct retinal cells by homeobox proteins. PLoS Biol. 2006; 4: e272.
55. Xia JH, Liu JX, Zhou L, Li Z, Gui JF. Apo-14 is required for digestive system organogenesis during fish embryogenesis and larval development. Int J Dev Biol. 2008; 52: 1089-98.

56. Choudhury M, Yamada S, Komatsu M, Kishimura H, Ando S. Homologue of mammalian apolipoprotein A-II in non-mammalian vertebrates. Acta Bioch Bioph Sin. 2009; 41: 370-8.

57. Zhang $\mathrm{T}$, Yao S, Wang $\mathrm{P}$, Yin $\mathrm{C}$, Xiao $\mathrm{C}$, Qian $\mathrm{M}$, et al. Apoa-II directs morphogenetic movements of zebrafish embryo by preventing chromosome fusion during nuclear division in yolk syncytial layer. J Biol Chem. 2011; 286: 9514-25.

58. Seth A, Machingo QJ, Fritz A, Shur BD. Core fucosylation is required for midline patterning during zebrafish development. Dev Dyn. 2010; 239: 3380-90.

59. Chen SR, Kimelman D. The role of the yolk syncytial layer in germ layer patterning in zebrafish. Development. 2000; 127: 4681-9.

60. Ebert AM, McAnelly CA, Srinivasan A, Linker JL, Horne WA, Garrity DM. $\mathrm{Ca}^{2+}$ channel-independent requirement for MAGUK family CACNB4 genes in initiation of zebrafish epiboly. Proc Natl Acad Sci USA. 2008; 105: 198-203.

61. Lachnit M, Kur E, Driever W. Alterations of the cytoskeleton in all three embryonic lineages contribute to the epiboly defect of Pou5f1/Oct4 deficient MZspg zebrafish embryos. Dev Biol. 2008; 315: 1-17.

62. Du S, Draper BW, Mione M, Moens CB, Bruce A. Differential regulation of epiboly initiation and progression by zebrafish Eomesodermin A. Dev Biol. 2012; 362: 11-23. 